\title{
A Satellite Method to Identify Structural Properties of Mesoscale Convective Systems Based on the Maximum Spatial Correlation Tracking Technique (MASCOTTE)
}

\author{
Leila M. V. CARVALho* \\ Department of Atmospheric Sciences, Institute of Astronomy and Geophysics, University of São Paulo, São Paulo, Brazil \\ Charles Jones \\ Institute for Computational Earth System Science, University of California, Santa Barbara, Santa Barbara, California
}

(Manuscript received 14 November 2000, in final form 29 January 2001)

\section{ABSTRACT}

\begin{abstract}
A simple, fully automated, and efficient method to determine the structural properties and evolution (tracking) of cloud shields of convective systems (CS) is described. The method, which is based on the maximum spatial correlation tracking technique (MASCOTTE), is a new alternative to the existent techniques available for studies that monitor the evolution of CS using satellite images. MASCOTTE provides as CS structural properties the following parameters: mean and variance of brightness temperature, horizontal area, perimeter, minimum brightness temperature, fractional convective area, center of gravity, and fragmentation. The fragmentation parameter has the potential to monitor the evolution of the CS. A new way of estimating the orientation and eccentricity of CS is proposed and is based on the empirical orthogonal function analysis of CS pixel coordinates. The method includes an accurate detection of splitting and merging of convective systems, which is a critical step in the automated satellite CS life cycle determination. Based on the magnitudes of the spatial correlation between consecutive satellite images and the changes in horizontal areas of CS, MASCOTTE provides a simple and skillful technique to track the evolution of CS life cycles. The MASCOTTE methodology is applied to infrared satellite images during seven consecutive days of the Wet-Season Atmospheric Mesoscale Campaign of the Large-Scale Biosphere-Atmosphere Experiment and ground validation experiment of the Tropical Rainfall Measuring Mission in the Brazilian state of Rondônia in the Amazon basin. The results indicate that MASCOTTE is a valuable approach to understanding the variability of CS.
\end{abstract}

\section{Introduction}

Convective systems (CS) in the earth's atmosphere occur in a wide spectra of time- and space scales and play important roles in the distribution of energy, momentum, and mass in the general circulation (Cotton and Anthes 1989). Furthermore, the associated precipitation is a key element in the hydrological cycle at regional to global scales. In particular, mesoscale convective systems (MCS) can be broadly defined as cloud systems associated with ensembles of thunderstorms with contiguous precipitation areas. Typically, their horizontal scales can extend $100 \mathrm{~km}$ or more in one direction, and their life cycles can last from a few

\footnotetext{
* Current affiliation: Institute for Computational Earth System Science, University of California, Santa Barbara, Santa Barbara, California.

Corresponding author address: Dr. Leila M. V. Carvalho, Institute of Astronomy and Geophysics, University of São Paulo, R. do Matão 1226, 05508-900 São Paulo, Brazil.

E-mail: leila@model.iag.usp.br
}

hours to as long as 2-3 days (Houze 1993). The observational description and theoretical understanding of these convective systems have grown steadily over the years, providing detailed conceptual models of the convective organization, three-dimensional wind circulation, thermal structure, precipitation, and microphysical processes occurring in different types of MCS (Houze 1977; Maddox 1980; Houze and Hobbs 1982; Mapes and Houze 1993). In this context, observational data with sufficient spatial resolution from operational networks of rawinsondes, meteorological radars, and field experiments have been fundamental, because they can resolve the genesis, maturity, and decay phases of MCS (Zipser 1988; Chen et al. 1996; Mohr and Zipser 1996).

The availability of satellite radiances has provided a unique way to monitor the evolution of CS (Cotton and Anthes 1989; Houze 1993), especially in regions that are strategically difficult to access such as open oceans and tropical rain forests. Furthermore, satellite methods that identify and track the structural properties of CS (e.g., mean brightness temperature, area, perimeter, and eccentricity) can provide valuable information 
on the dynamical mechanisms involved in their life cycle, including the interaction between CS and the large-scale circulation (Silva Dias and Ferreira 1992; Cohen et al. 1995). Many previous satellite-tracking methods have been proposed, which include subjective and automated methods (Velasco and Fritsch 1987; Williams and Houze 1987; Machado et al. 1998). The reader is referred to Machado et al. (1998) for a thorough review on the state-of-the-art satellite tracking techniques. Automated methods, to take a single instance, can be useful for extensive meteorological field campaigns when tracking of large satellite imagery databases needs to be performed, sometimes even in realtime monitoring. Although the satellite methods that describe structural properties of CS can rely on the same kind of data, that is, infrared and/or visible and microwave satellite radiances, significant differences may exist in the methodology that tracks the evolution of CS. For instance, the CS life cycle obtained from satellite tracking methods is one parameter that can contaminate cloud cluster statistics. As will be demonstrated, the accurate determination of splitting and merging of CS cloud shields is a crucial requirement in this process. Therefore, it is important to compare different techniques so that automated methods provide reliable and consistent results.

The purpose of this paper is to introduce a simple and efficient automated method to track and characterize structural properties of CS cloud shield simultaneously using satellite infrared (IR) images. The approach is based on the maximum spatial correlation tracking technique (MASCOTTE). We show how MASCOTTE specifically allows the characterization of splitting and merging of CS as they occur during their life cycles. We compare different methods to identify the horizontal axis of CS cold-top orientation. Some structural parameters of clouds such as eccentricity and fragmentation are used in the identification of different stages of intensification and decaying of CS. As an application of MASCOTTE, we focus our attention on seven consecutive days during the Wet-Season Atmospheric Mesoscale Campaign (WETAMC) of the Large-Scale Biosphere-Atmosphere Experiment (LBA) (Nobre et al. 1996; Silva Dias et al. 2000). This field experiment, which was realized from December of 1998 through February of 1999 in the Brazilian state of Rondônia in the Amazon basin, was a joint scientific venture with the ground validation component of the Tropical Rainfall Measuring Mission (TRMM). The paper is organized as follows. The description of the method is presented in section 2. Section 3 discusses different techniques to determine properties of the life cycle of CS and presents some structural properties of CS during the case studies. Section 4 discusses the application of the method for the characterization of the time evolution of CS. Section 5 summarizes the methodology and our main conclusions.

\section{Maximum spatial correlation tracking technique}

The basic approach of our satellite tracking methodology and the characterization of structural properties of CS is now described. The method uses as input fields brightness temperature from satellite images. In this work, Geostationary Operational Environmental Satellite $(G O E S-8)$ images were used. These images have horizontal resolution of $4 \mathrm{~km}$ and are part of the TRMM-LBA program and at the time of writing were available online from the National Aeronautics and Space Administration (NASA) Goddard Space Flight Center at http://lake.nascom.nasa.gov/data/TRMM. In addition, the high temporal frequency of GOES-8 images, typically every $30 \mathrm{~min}$, allows an adequate resolution of the different stages of development of convective systems. In the discussion that follows, hourly satellite images were used. However, as will be explained later, an important advantage of our method is that it characterizes the correct evolution of CS (e.g., splitting or merging of systems) even if the time interval between images is $3 \mathrm{~h}$.

The use of satellite infrared images, that is, brightness temperature $T_{B}$, usually requires an arbitrary choice of threshold to identify regions of convection. There is general agreement that $T_{B}$ values below a threshold of $245 \mathrm{~K}$ satisfactorily identify convective systems (Velasco and Fritch 1987; Mapes and Houze 1993; Miller and Fritch 1991; Machado and Rossow 1993; Machado et al. 1998). The reasoning in the threshold selection is that a buoyant parcel in the Tropics is likely to originate below the 700-hPa level to reach the level of $T_{B}<245$ $\mathrm{K}$, which ensures the existence of deep convection (Machado et al. 1998). In addition, the near-linear dependence between the convective system area and its threshold indicates the insensitivity to the choice of a specific value in a range of 10-20 K (Mapes and Houze 1993; Machado et al. 1992). Garreaud and Wallace (1997), for instance, used fraction of clouds with $T_{B} \leq 235 \mathrm{~K}$ with $0.5^{\circ}$ lat $\times 0.5^{\circ}$ long resolution and 3 - $\mathrm{h}$ temporal resolution to estimate the diurnal march of convective cloudiness over the tropical and subtropical Americas. Because MASCOTTE is applied here over tropical South America, convective systems are identified in the satellite images whenever regions of $T_{B}$ are less than or equal to $235 \mathrm{~K}$ to be consistent with Garreaud and Wallace (1997). For the WETAMC LBA-TRMM campaign, cloud tops with $T_{B} \leq 235 \mathrm{~K}$ were in general near the level of $250 \mathrm{hPa}$. With this threshold in mind, we investigate mostly anvil regions and the embedded areas of active deep convection (Johnson et al. 1990). Note that, given the versatility of MASCOTTE, the threshold value can be adapted to other specific regions.

The definition of CS based on the temperature threshold technique allows wide ranges of horizontal areas. Machado et al. (1998) showed positive correlations between the lifetime of CS and their sizes. Their results indicate that, in general, CS that reach equivalent radius 
of $100 \mathrm{~km}$ or less and temperature thresholds of $245 \mathrm{~K}$ are mostly associated with lifetimes shorter than $3 \mathrm{~h}$. To test the skill of our method and emphasize the tracking of CS with long life cycles, we consider systems with equivalent radius $R \geq 100 \mathrm{~km}$, the same threshold of Machado et al. (1998). These limits of brightness temperature and horizontal area in our methodology imply that the life cycle of a given CS begins when the convective system has $T_{B} \leq 235 \mathrm{~K}$ and $R \geq 100 \mathrm{~km}$. The end of the life cycle, on the other hand, occurs when $T_{B}>235 \mathrm{~K}$ or $R<100 \mathrm{~km}$. The combination of both criteria (i.e., $T_{B} \leq 235 \mathrm{~K}$ and $R \geq 100 \mathrm{~km}$ ) is hereinafter referred to as $T-R$ criteria.

The MASCOTTE basic hypothesis is that the spatial correlation between regions defined by two different cloud systems in successive images is a simple and powerful method to identify the evolution of spatial patterns associated with CS. Similar techniques have been applied to estimate tropospheric winds based on satellite cloud displacements (Endlich and Wolf 1981). The basic steps involved in the MASCOTTE method are as follows.

1) We consider that $N$ satellite images of $T_{B}$ are available with time interval $\Delta t$. In this work, $\Delta t$ is taken to be $1 \mathrm{~h}$. However, as will be shown later, the method is successful for $\Delta t$ intervals up to $3 \mathrm{~h}$. For each satellite image at time $t_{i}, 0 \leq i \leq N$, regions of CS are identified according to the $T-R$ criteria. Regions that do not meet the threshold criteria are set to null value. This procedure allows a large difference between clouds and the background mask, thus emphasizing the overlapping of systems. We consider that, at time $t_{i}$, there are $m$ CS identified.

2) At time $t_{i}$, the $k$ th $\mathrm{CS}(1 \leq k \leq m)$ is first isolated in the image (i.e., the remainder of the image is set to the background mask value), which has a size of $I_{C \times L}$, where $C$ and $L$ are the number of pixels in the east-west and north-south directions, respectively. For the application to the TRMM-LBA experiment discussed in this paper, the 4-km satellite image has $C=1332$ by $L=860$ pixels. We then consider the next satellite image at time $t_{i+1}$ and identify all the $\mathrm{CS}$. The CS at $t_{i+1}$ that has maximum spatial correlation $\left(r_{s}>0.30\right)$ with the $k$ th $\mathrm{CS}$ at $t_{i}$ is considered the new spatial position of the CS. The threshold value of $r_{s}>0.30$ was determined from many tests performed in the initial development of the algorithm. This value ensures reliable tracking of CS using satellite images with a 1-h time interval. The process is repeated for all CS at time $t_{i}$ and starts again for the next satellite image.

3) For each time $t_{i}$ and each CS, structural properties are computed. These include the horizontal area $A$, perimeter $P$, mean and variance of $T_{B}$, minimum $T_{B}$, and fractional convective area $F C\left[=100\left(A_{T C} / A\right)\right]$, where $A_{T C}$ is the area within the CS such that $T_{B} \leq$ $210 \mathrm{~K}$. According to rawinsondes during the WE-
TAMC LBA-TRMM campaign, $T_{B} \leq 210 \mathrm{~K}$ was associated with cloud tops that reached heights above $13 \mathrm{~km}$ in an environment in which the tropopause was very often above $16.0 \mathrm{~km}$. Furthermore, the $T_{B}$ $\leq 213-208 \mathrm{~K}$ threshold has been identified with precipitation in the Tropics (Williams and Houze 1987; Mapes and Houze 1993; Rickenbach 1999) and is therefore of interest for the monitoring of CS life cycles. In addition, the spatial coordinates of the center of gravity $\left(X_{\mathrm{CG}}, Y_{\mathrm{CG}}\right)$ are defined as the $T_{B}$-weighted average:

$$
X_{\mathrm{CG}}=\frac{\sum_{i=1}^{N_{P}} X_{i} T_{B i}}{\sum_{i=1}^{N_{P}} T_{B i}} \quad Y_{\mathrm{CG}}=\frac{\sum_{i=1}^{N_{P}} Y_{i} T_{B i}}{\sum_{i=1}^{N_{P}} T_{B i}},
$$

where $X_{i}$, and $Y_{i}$ are the CS pixel coordinates in the east-west and north-south directions, respectively, and the summation is carried out over all $N_{P}$ pixels within the given $\mathrm{CS}$.

4) The orientation of the CS cloud shield is computed in two ways. First, a straight line is fitted to the $\left(X_{i}\right.$, $Y_{i}, i=1, N_{P}$ ) pixel coordinates by a least squares criterion and the counterclockwise angle between the east-west direction and the straight line is recorded. However, as will be discussed later, a satellite method that relies on this definition of orientation can oftentimes obtain misleading results, depending on the geometrical structure of the CS. In contrast, a new way of computing the orientation is proposed. We consider a given CS in which the array of pixel coordinates is given by $\left(X_{i}, Y_{i}, i=1, N_{P}\right)$. The means $\bar{X}$ and $\bar{Y}$ are first subtracted from the vectors $X_{i}$ and $Y_{i}$ and an empirical orthogonal function (EOF) analysis is computed on the covariance matrix determined by $X^{\prime}$ and $Y^{\prime}$ perturbations [see Jackson (1991) for details on EOF analysis]. The result of this operation is a pair of eigenvalues and eigenvectors that explains the total variance of the geographical coordinates of the given CS. A second orientation is then computed as the counterclockwise angle between the east-west direction and the direction of the first eigenvector. This way of computing the orientation of the CS has the advantage that the first eigenvector maximizes the spatial variance of the CS cloud shield. Furthermore, another important property to characterize the structure of CS is the eccentricity, which in our method is defined by the ratio of the norms $\|$ EOF2 $\|/\|$ EOF1 1 , where EOF2 and EOF1 are the second and first eigenvectors, respectively.

Oftentimes, as the CS evolves, splitting of cloud features may happen when changes occur in the precipitation pattern and three-dimensional wind structure. This process can be indicative of intensification of precipitation at some point and decaying of part of the 
upward movement of the storm (Cotton and Anthes 1989). However, the propagation of the storm may continue under some favorable environmental conditions (Houze 1993). The identification of splitting during the life cycle is important for the monitoring of dynamical properties of CS (Houze 1993). The splitting of a CS at time $t_{i}$ is identified by MASCOTTE when more than one CS with positive and high spatial correlation is observed at time $t_{i+1}$. As will be shown with examples, the horizontal area of the system decreases. MASCOTTE continues the tracking of CS with the assumption that the propagation is now defined by the most correlated CS in subsequent satellite images. MASCOTTE also maps the position of any part of the splitting CS to follow its life cycle until it decays, which usually happens in a short period. Furthermore, because of the definition of CS using the $T_{B}$ threshold, merging of high clouds is also frequently observed, which is in fact related to the connection of anvil regions. At this point, it is difficult to identify the main system because its life cycle is determined by the interaction of more than one $\mathrm{CS}$. However, the merging is characterized in MASCOTTE by positive spatial correlation, because the identity of a tracked CS is kept as it merges with another one. These situations can be clearly identified during the life cycle when the spatial correlation decreases and the horizontal area increases between two consecutive satellite images.

Our inspection of 150 merges throughout different CS life cycles during the WETAMC cases considered here indicates that a reasonable criterion for the identification of merges is the increase of area followed by a decrease in $r_{s}$ of more than $10 \%$ of the previous value. The resulting shape of a CS is substantially modified after merges because of the introduction of new systems with different sizes and forms. When the increase in area is only due to expansion of the original CS, $r_{s}$ increases in the majority of the cases. MASCOTTE can also identify merges and splits that eventually happen simultaneously, as long as the CS maintains part of its spatial characteristics in two consecutive images. Oftentimes, however, CS can merge with systems that have equivalent radius less than $100 \mathrm{~km}$. Other times, CS show a decrease in area due to either decaying of the system (sinking of cloud tops) or splitting of cells with $R$ less than $100 \mathrm{~km}$. A posteriori refinement in the algorithm is applied to identify these dynamical processes. Our assumption is that these dynamical variations of the $\mathrm{CS}$ are associated with rapid changes in structural properties. An analysis performed with 100 life cycles with duration longer than $3 \mathrm{~h}$ (not shown) indicated that large accelerations are observed mainly in association with large rates of change of EOF1 orientation, perimeter, and direction. These results have been used to improve the MASCOTTE algorithm with the implementation of flags to indicate merges and splits that cannot be promptly detected with the $T-R$ criteria. One advantage of this detailed monitoring is that it provides enough infor- mation to reconstruct the entire life cycle of any CS, including interactions with other systems.

In summary, MASCOTTE yields the following structural properties for the $T_{B}$ and $R$ thresholds considered: average duration of the CS, total number of systems, and number of splits and merges. For the life cycle of individual CS, MASCOTTE determines area (Area), mean $\left(T_{\text {mean }}\right)$, and variance (Var) of brightness temperatures; minimum brightness temperature $\left(T_{\min }\right)$; eccentricity (Ecc); orientation of CS cold tops based on the first EOF orientation (EOF1) and least squares (LS) fit; and fraction of cold tops with $T_{B} \leq 210 \mathrm{~K}(\mathrm{Fc})$. MASCOTTE additionally determines the speed of displacement of CS center of gravity $(V)$, direction of propagation (DD), and fragmentation (Fluct) resulting from both dynamical and physical processes (explained in sections 3 and 4).

To put into context, we briefly summarize the main MASCOTTE characteristics by comparing them with the Machado et al. (1998) automated method. That method is very well documented and provides a framework for an objective discussion about the skill of a satellite tracking technique. Table 1 indicates MASCOTTE structural parameters and the correspondence with the Machado et al. (1998) procedure. Although some properties are common to both methods, note the differences in the tracking approaches. For instance, to track CS, the Machado et al. (1998) automated method assumes circular geometries for the systems overlapping and uses the weighted average of 28 structural properties. In contrast, the only requirement for MASCOTTE to determine the temporal evolution of CS is a maximum spatial correlation greater than a specified threshold. The main differences regarding structural parameters are the orientation of cloud tops and, consequently, eccentricity, convective fraction, perimeter and fragmentation, detection of merging and splits, and propagation characteristics. MASCOTTE does not compute number of convective clusters (contiguous region of brightness temperature below a given threshold) or properties associated with the largest convective cluster embedded in the CS. The reasoning is that structural parameters computed for $160 \mathrm{CS}$ over the Tropical Pacific Ocean indicated a linear correlation $(0.93)$ between $R$ and number of convective clusters $\left(T_{B}<218 \mathrm{~K}\right)$ and between $R$ and the largest convective cluster (0.85) but practically no correlation (0.16) between $R$ and Fc $\left(T_{B}\right.$ $<218 \mathrm{~K}$ ) (Carvalho 1998). For the sake of simplicity and to avoid redundancies, the MASCOTTE variable representative of the enhancement of the convective area of the CS is restricted to $\mathrm{Fc}\left(T_{B} \leq 210 \mathrm{~K}\right)$.

\section{Temporal and structural properties of CS using MASCOTTE}

To demonstrate the application of MASCOTTE, we selected as a case study seven consecutive days from 12 to 18 February 1999 during the WETAMC LBA- 
TABLE 1. Parameters determined with MASCOTTE and the automated method of Machado et al. (1998).

\begin{tabular}{|c|c|c|}
\hline Parameter & MASCOTTE & Machado et al. (1998) \\
\hline CS center of gravity & Yes $\left(T_{B} \leq 235 \mathrm{~K}\right)$ & Yes $\left(T_{B} \leq 245 \mathrm{~K}\right)$ \\
\hline Radius of CS & Yes & Yes \\
\hline Average CS $T_{B}$ & Yes & Yes \\
\hline Minimum $T_{B}$ & Yes & Yes \\
\hline Variance of CS $T_{B}$ & Yes & Yes \\
\hline Gradient of $T_{B}$ & No & Yes \\
\hline Eccentricity of $\mathrm{CS}$ & Yes & Yes \\
\hline Convective fraction & Yes $\left(T_{B} \leq 210 \mathrm{~K}\right)$ & Yes $\left(T_{B} \leq 218 \mathrm{~K}\right)$ \\
\hline Orientation of cloud tops: least squares & Yes & Yes \\
\hline Orientation of cloud tops: EOF1 & Yes & No \\
\hline Speed of CS propagation & Yes & Yes \\
\hline Direction of propagation & Yes & Yes \\
\hline Perimeter and fragmentation & Yes & No \\
\hline Identification of total splits during life cycles & Yes & No \\
\hline Identification of life cycle end due to merging & Yes & No \\
\hline Flags for splitting/sinking and merges with CS that do no satisfy the $T-R$ criteria & Yes & No \\
\hline No. of convective top (CT) & No & Yes $\left(T_{B} \leq 218 \mathrm{~K}\right)$ \\
\hline Area, location, and propagation direction and speed of the largest CT & No & Yes \\
\hline
\end{tabular}

TRMM experiment. The purpose of this selection is the continuous availability of satellite images with $1 \mathrm{~h}$ of time interval. We emphasize that the main goal of this paper is to present the automated applicability of MASCOTTE for an extensive field experiment campaign. The satellite surveillance area extends from $12.5^{\circ} \mathrm{N}$ to $18.5^{\circ} \mathrm{S}$ and from $34.0^{\circ}$ to $82.0^{\circ} \mathrm{W}$. The large spatial coverage of the satellite methodology provides propagation characteristics of CS not only over Rondônia state but also over the entire tropical South America. This coverage has important implications for investigating the organization of CS in the Amazon and possible interactions with the South Atlantic convergence zone and the intertropical convergence zone.

\section{a. Monitoring of CS life cycles in the Amazon basin}

The application of MASCOTTE is now discussed in detail. Some important aspects about MASCOTTE for the determination of CS life cycles are summarized in Table 2. These aspects include the total number of CS and CS life cycles, splits, and merges observed in the period that satisfied the $T-R$ criteria. Also indicated is the total number of flags that were likely associated with splitting/sinking or merging with CS that did not satisfy the $T-R$ criteria. The results shown in Table 2 are indicative of the importance of the correct determination of merges and splits during CS life cycles. Approxi- mately $30 \%$ of CS life cycles ended up merging with other CS that satisfy the $T-R$ criteria. Meanwhile, $8 \%$ of CS split during their life cycle and resulted in two or more CS that match the $T-R$ criteria. The fraction of merges is $10 \%$ higher than what was estimated in Machado et al. (1998) for a 3-h time interval, whereas the fraction of splits follows approximately the same rate observed in their study. However, if one considers the possibility of splitting (or sinking) of CS that do not satisfy $T-R$ criteria, the rate of splits increases to approximately $21 \%$.

The critical issue of accurately detecting CS splitting/ merging in an automated satellite tracking technique is discussed in the next results. The impact on the CS life cycles from the inclusion of merging with CS satisfying the $T-R$ criteria is elucidated in Fig. 1. For the period of 12-18 February, we first considered the life cycles of all CS that satisfy the $T-R$ criteria regardless of whether merges had occurred. The resulting distribution is shown as a function of life cycle duration (black bars). Second, the life cycles were recomputed using the MASCOTTE detection of merges. In that case, whenever the CS merged with another CS that satisfied the $T-R$ criteria, the life cycle ended (dotted bars). When merges are considered as part of a life cycle, the tail of the distribution increases toward the longer values and the number of life cycles in the shorter categories decreases as expected. Two life cycles of $59 \mathrm{~h}$ were observed when

TABLE 2. MASCOTTE summary during 12-18 Feb: total number of CS that satisfy the $T-R$ criteria, total number of life cycles, number of life cycles that end up merging, and number of splitting/sinking and merging flags in association with systems that do not satisfy the $T$ $R$ criteria. One-hour time interval between images is considered.

\begin{tabular}{ll}
\hline \hline Total CS & 1724 \\
Total life cycles & 431 \\
Life cycles with merging (CS satisfy the $T-R$ criteria) & 129 (30\% of life cycles) \\
Splitting during life cycles (CS satisfy the $T-R$ criteria) & 133 (8\% of total CS) \\
Total flags for merging (CS do not satisfy $T-R$ criteria) & 186 (11\% of total CS) \\
Total flags for splitting/sinking (CS do not satisfy $T-R$ criteria) & 226 (13\% of total CS) \\
\hline
\end{tabular}




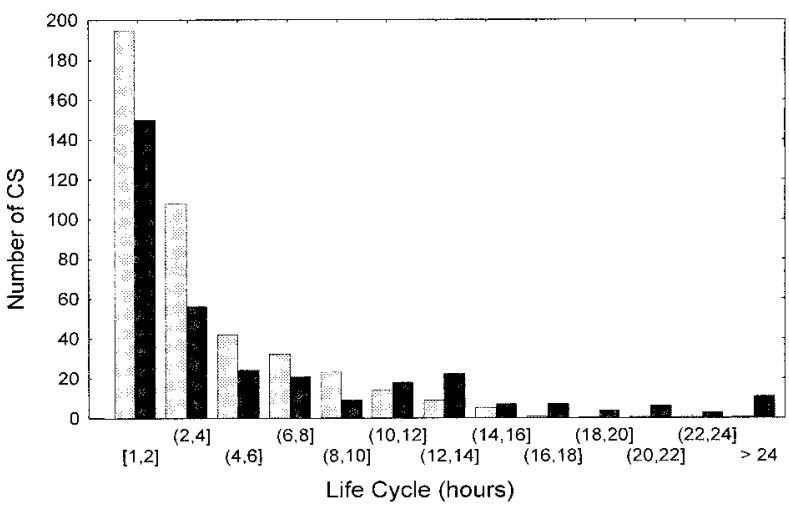

FIG. 1. Distribution of CS life cycles for 12-18 Feb 1999. Black bars denote life cycles of all CS that satisfy the $T-R$ criteria regardless of whether merges had occurred. Dotted bars indicate life cycles excluding merges.

merges were considered as part of the life cycle. On the other hand, when merges are not considered as part of the life cycle, the longest duration was on the order of 1 day $(23 \mathrm{~h})$. The observation of a larger number of CS with life cycles longer than $24 \mathrm{~h}$ in the Machado et al. (1998) automated tracking relative to semiautomated is likely related to the nonobjective criterion for the decision about whether merges should be included in a given life cycle (see Fig. 2 in Machado et al. 1998).

Another relevant implication of the objective determination of merges and splits is concerned with the estimation of CS speed and direction of propagation. During CS splitting events, a sudden shift in the position of the center of gravity is observed. As a consequence, speeds of more than $250 \mathrm{~km} \mathrm{~h}^{-1}$ are oftentimes recorded. They are, of course, an estimate of the large displacement of the CS center of gravity toward the position of one of the splitting CS. Likewise, during merges, a substantial modification of the form of the CS leads to a large displacement of the center of gravity. Any estimation of propagation speed during the occurrence of merges and splits is therefore an unrealistic approach of the actual velocity of propagation of the CS. Nonetheless, splits occur very often and can be considered as part of a life cycle. A method that relies on the criterion of speed of displacement of center of gravity to decide about a sequence in a life cycle can be potentially misleading.

The effect on the estimation of CS displacement speed and direction with inclusion of flags for CS splitting/ merging that does not not satisfy the $T-R$ criteria is illustrated in Fig. 2. Figure 2a shows the distribution of CS displacement speed in the following categories: 1) only nonflagged CS (black bars) and 2) all CS within a life cycle (dotted bars). We recall that no splitting or merging with systems that satisfy $T-R$ criteria is considered in this distribution. Nonetheless, the range of speeds is noticeably larger when splitting and merges are not properly flagged. Speeds on the order of 500
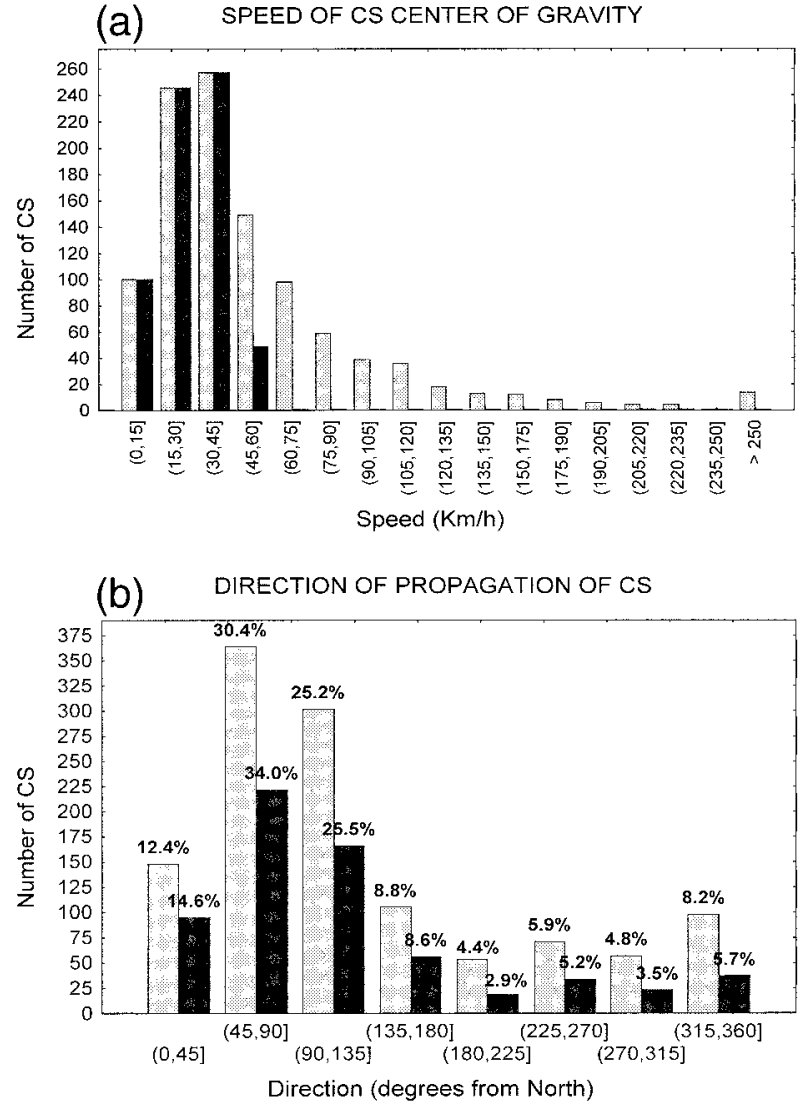

FIG. 2. Distribution of (a) speed and (b) direction of CS propagation for all events (dotted bars) and excluding events flagged for splitting/ merges (black bars). Splitting resulting in CS that match the $T-R$ criteria are excluded in both categories.

$\mathrm{km} \mathrm{h}^{-1}$ or more can be computed in some cases, which is a consequence of rapid changes in the form of the CS during these events. This range of magnitudes is greater than the $60 \mathrm{~m} \mathrm{~s}^{-1}\left(216 \mathrm{~km} \mathrm{~h}^{-1}\right)$ maximum criterion accepted for searching life cycles in the Machado et al. (1998) automated tracking. Therefore, based only on this criterion, a large majority of flagged CS life cycles determined with MASCOTTE would be potentially accepted as satisfying the Machado et al. (1998) automated tracking method. We recall that MASCOTTE does include flagged events as part of a life cycle, but speeds are only computed whenever splits resulting in $\mathrm{CS}$ that match the $T-R$ criteria are not occurring. Likewise, the implication of flagged events for the determination of propagation direction is shown in Fig. 2b. This distribution indicates a decrease in the number of CS in each category, although the form of the distribution remains approximately the same. Details on the MASCOTTE flagged CS will be further discussed in section 4.

The CS life cycle is considered with MASCOTTE, in sum, as the period that a CS is observed that matches the $T-R$ criteria. The end of a life cycle is also deter- 
(a)

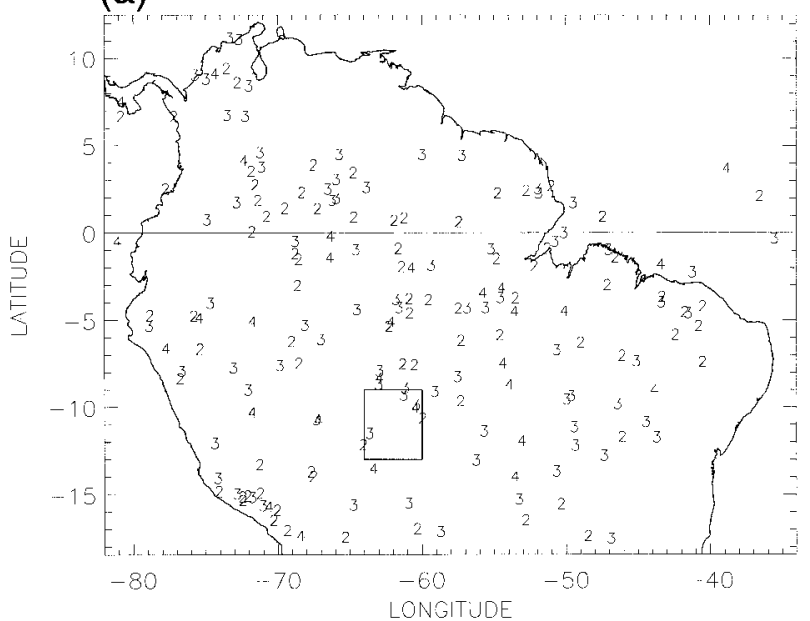

(c)

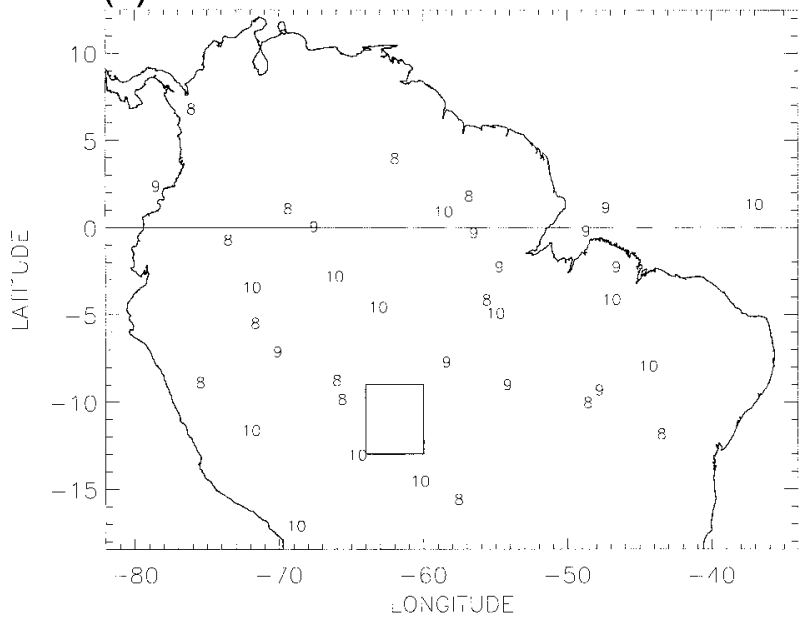

(b)

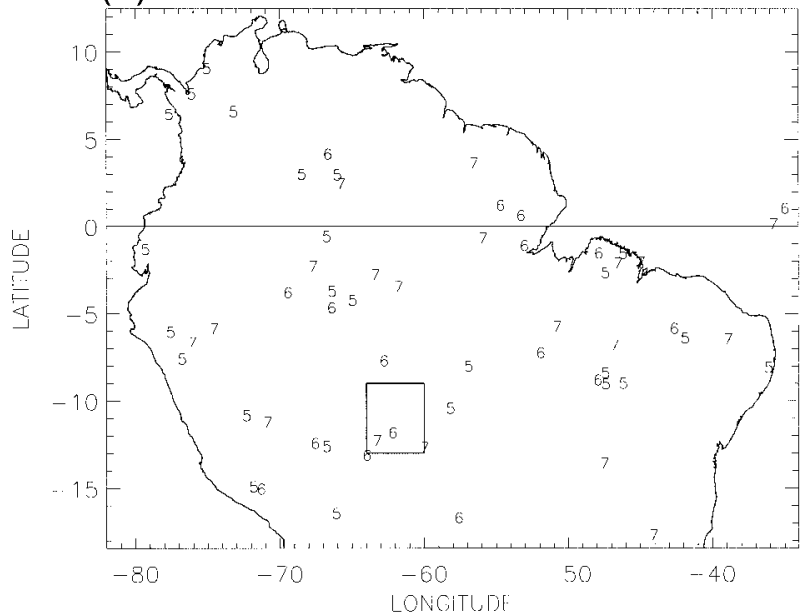

(d)

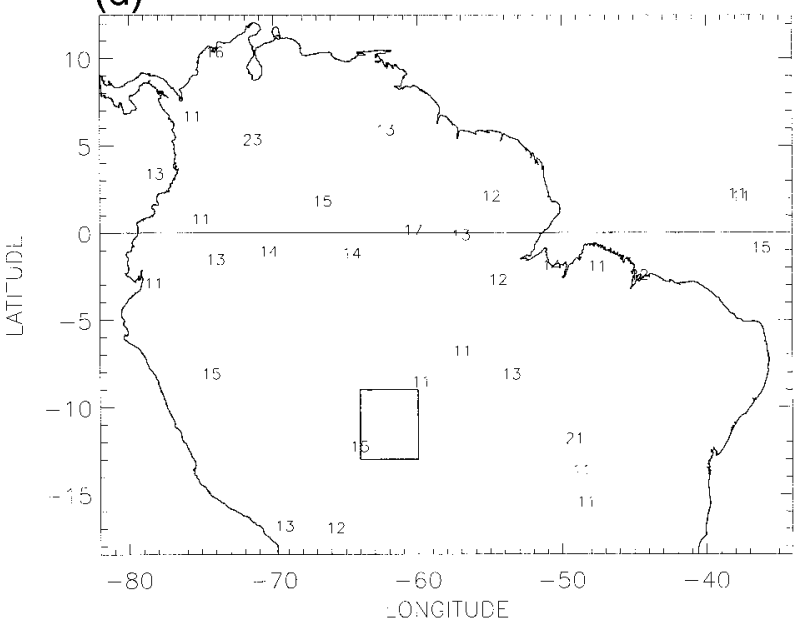

FIG. 3. Origin of CS in four categories: (a) 2-4, (b) 5-7, (c) 8-10, and (d) 10-24 h for 12-18 Feb 1999 . CS life cycle (h) is indicated at the position of the CS origin. The black square in the lower part of the figures indicates the domain of the Tropical Oceans and Global Atmosphere and S-band Doppler dual polarization radars in the Brazilian state of Rondônia during the WETAMC LBA-TRMM field campaign.

mined when a merge with another CS that matches the $T-R$ criteria occurs. Figure 3 shows the spatial distribution of the origin of the CS according to the life cycle (hours). The origin of CS with life cycles less than or equal to $4 \mathrm{~h}$ is well distributed in the area, with no clear geographic preference (Fig. 3a). Although the case study is based on 7 days, as the life cycle increases to periods between 5 and $7 \mathrm{~h}$, a pattern of parallel bands aligned northwest-southeast becomes more suggestive (Fig. 3b). Garreaud and Wallace (1997), using composites of IR images with $T_{B} \leq 235 \mathrm{~K}$, found two parallel bands (2000 km long and about $400 \mathrm{~km}$ wide) of maximum convective cloudiness with the same orientation during the wet season (December-February). They consider this banded pattern to be a systematic feature over South America (see their Fig. 3). Special attention should be given to the band of CS with origin near the northnortheast coast of South America that exhibits life cy- cles between 5 and $7 \mathrm{~h}$. The origins of CS with shorter life cycles (less than $5 \mathrm{~h}$ ) seem to be displaced offshore. This feature was also examined in Garreaud and Wallace (1997) in association with strong diurnal variability. As the life cycle increases to $8-10 \mathrm{~h}$ (Fig. 3c), fewer CS origins were observed in north-northeast coastal South America, though the northwest-southeast-oriented pattern remains suggestive. Origins of life cycles longer than $10 \mathrm{~h}$ are more concentrated above $5^{\circ} \mathrm{S}$ during the current case studies (Fig. 3d).

The relationship between the CS onset period (i.e., time when the CS that matched the $T-R$ criteria was first observed) and the life cycle is investigated in Fig. 4. The onset period is divided in four categories: evening to predawn (Ev-Dw, from 0045 to 0545 UTC), predawn to morning (Dw-Mo, from 0645 to 1145 UTC), morning to afternoon (Mo-Af, from 1245 to 1745 UTC), and afternoon to evening (Af-Ev, from 1845 to 2345 UTC). 


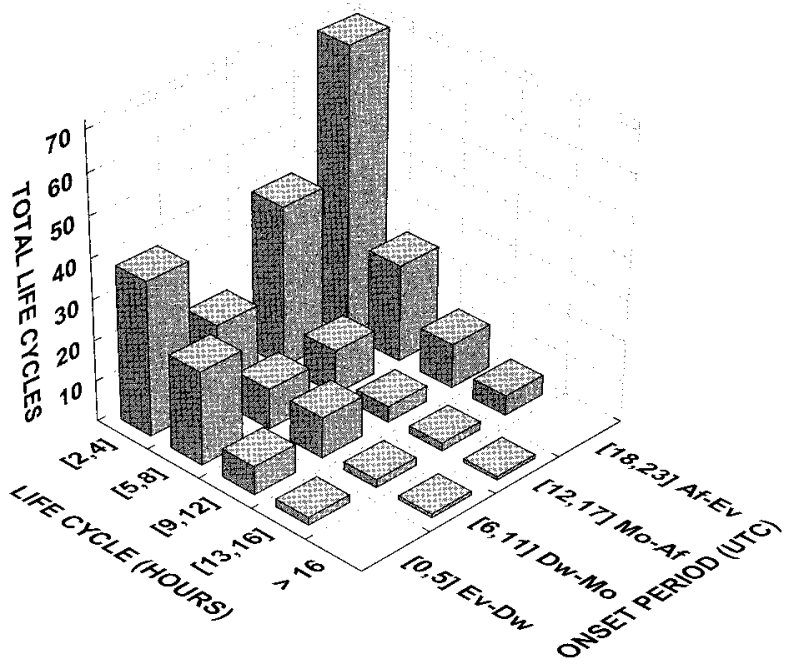

FIG. 4. Bidimensional distribution of life cycle and CS onset period (see text for details). Onset period refers to 0045-0545 UTC (EvDw: evening to predawn), 0645-1145 UTC (Dw-Mo: predawn to morning), 1245-1745 UTC (Mo-Af: morning to afternoon), and 1845-2345 UTC (Af-Ev: afternoon to evening).

It is clear that the maximum daily heating plays an important role for the relatively short-lived CS (shorter than $5 \mathrm{~h}$ ). The maximum during the Af-Ev period is about 2 times larger than during the Ev-Dw and MoAf periods. Nonetheless, the approximately equal contribution of onsets during the Mo-Af and Ev-Dw indicates more than the gradual decay during night hours suggested in Garreaud and Wallace (1997). To a certain extent, it represents the origin of new CS that have a preferable nocturnal life cycle. Longer life cycles (5-8 h) also appear to be a characteristic of the nocturnal propagating CS. However, investigations with more case studies would be necessary to address properly the pattern shown in Fig. 4 for long life cycles.

The relationships between life cycles and horizontal dimensions of the CS can be further investigated with MASCOTTE results. Because the variability of the CS horizontal dimensions is larger during long life cycles, the correspondence between life cycles and sizes of CS is appropriately characterized by the CS maximum radius (Rmax). The bidimensional distribution of Rmax and respective life cycle is indicated in Fig. 5. For 100 $\leq R \max \leq 200 \mathrm{~km}$, there seems to be a positive correlation between Rmax and life cycle, as suggested in Machado et al. (1998) with the use of mean radius (Rmean). For large values of Rmax, however, there is no evidence of this dependence. The evenly distributed events for Rmax of greater than $200 \mathrm{~km}$ are possibly associated with merges of CS that satisfy $T-R$ criteria. During the occurrence of merges, the equivalent radius is oftentimes the Rmax of a life cycle. Because the resulting CS is considered in a separated life cycle, they are not necessarily following the linear tendency observed for short periods.

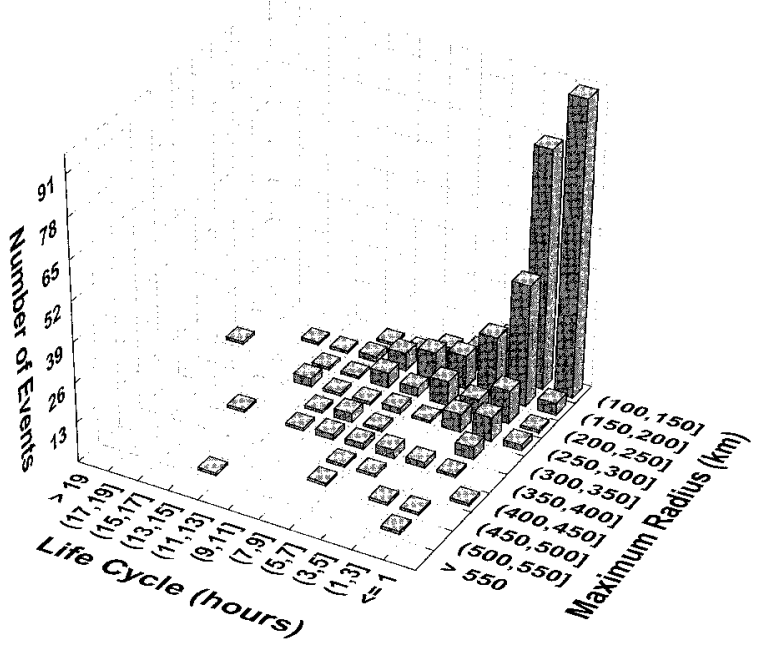

FIG. 5. Bidimensional distribution of $\mathrm{CS}$ maximum radius $(\mathrm{km})$ and respective life cycle $(\mathrm{h})$

The diurnal variation of the number of CS from 12 to 18 February is shown in Fig. 6. Time is displayed for local time in Rondônia, Brazil. The late afternoon maximum with gradual decrease during the night and minimum in late morning is consistent with the observations of Garreaud and Wallace (1997). Figure 6, similar to Garreaud and Wallace (1997) IR daily composites, does not provide any information on the onset of CS and their life cycle. To obtain a more comprehensive overview of the CS diurnal variability, Fig. 7 combines CS life cycle duration, onset time interval, and displacement. For each CS, the information is plotted in the origin of the life cycle ( $\mathrm{x}$ symbol), the duration (hours), and displacement (line segment). Note the difference between trajectory and displacement adopted here. Trajectories of the center of gravity of CS can sometimes be very irregular. The CS displacement shown in Fig. 7 indicates the difference between the position at the beginning (x symbol) and end of a life cycle. It can, however, give an idea of the direction of propagation of the CS. We focused our analysis on the period of increasing and decreasing number of CS indicated in Fig. 6. To emphasize the contrast between the two periods, only CS with life cycles of $\geq 4 \mathrm{~h}$ are

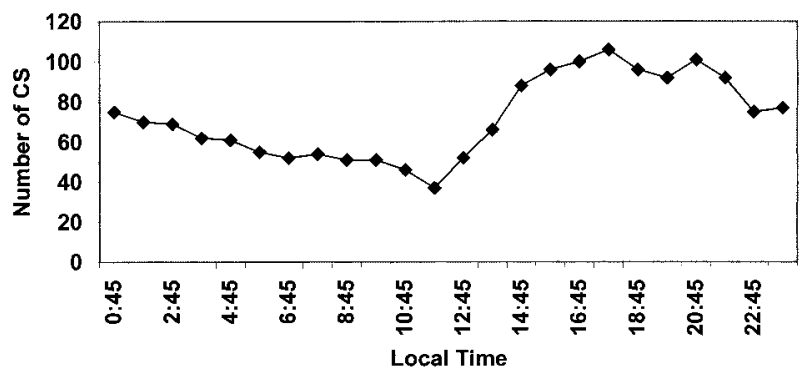

FIG. 6. Diurnal variation of CS $12-18$ Feb that satisfy the $T-R$ criteria. Local time refers to Rondônia, Brazil (UTC $-4 \mathrm{~h}$ ). 

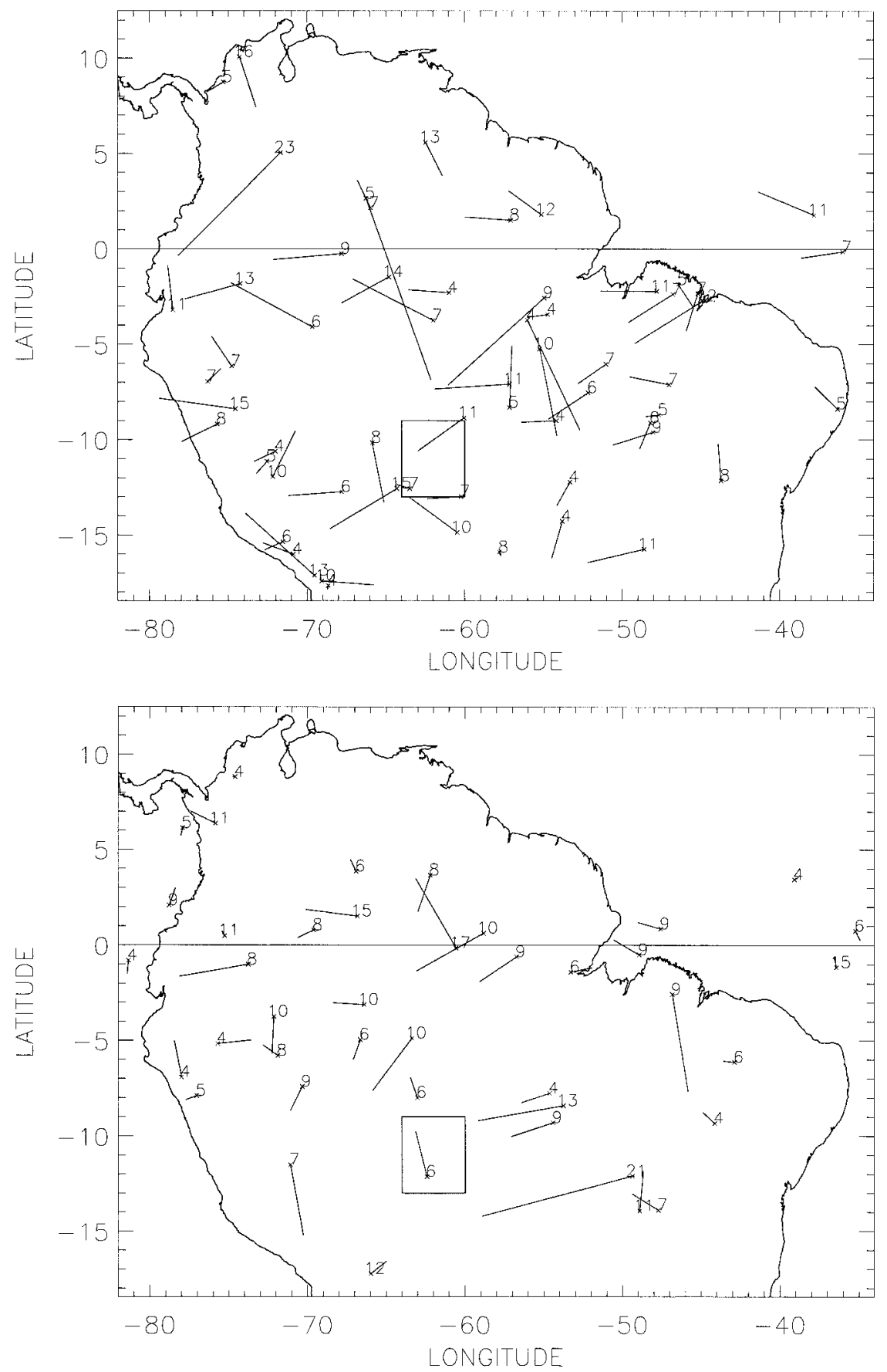

FIG. 7. Origin (x), displacement (lines), and life cycle (h) of the CS in the (top) afternoon (1245-1845 LT) and (bottom) early morning (0045-0645 LT).

shown in Fig. 7. There are $30 \%$ more CS with life cycles of $\geq 4 \mathrm{~h}$ in the afternoon (total $=60$ life cycles) than at night (total $=42$ life cycles). However, $47 \%$ of CS with onset in the afternoon showed life cycles of $\geq 8 \mathrm{~h}$, whereas this rate increases to $57 \%$ for the nocturnal CS. Some relevant characteristics of the diurnal variation of $\mathrm{CS}$ are the afternoon maximum in the north-northeast and western South America coast, in contrast with the nocturnal inland origin of CS. Note the suggestive increase of offshore CS during the night. The nocturnal peak of large and long-living oceanic CS has been observed in the tropical Pacific Ocean during active periods of convection (Chen and Houze 1997). The authors observed that these systems begin late in the afternoon and continue during the night, when they have their largest dimension. Although the $T-R$ criteria do not al- 


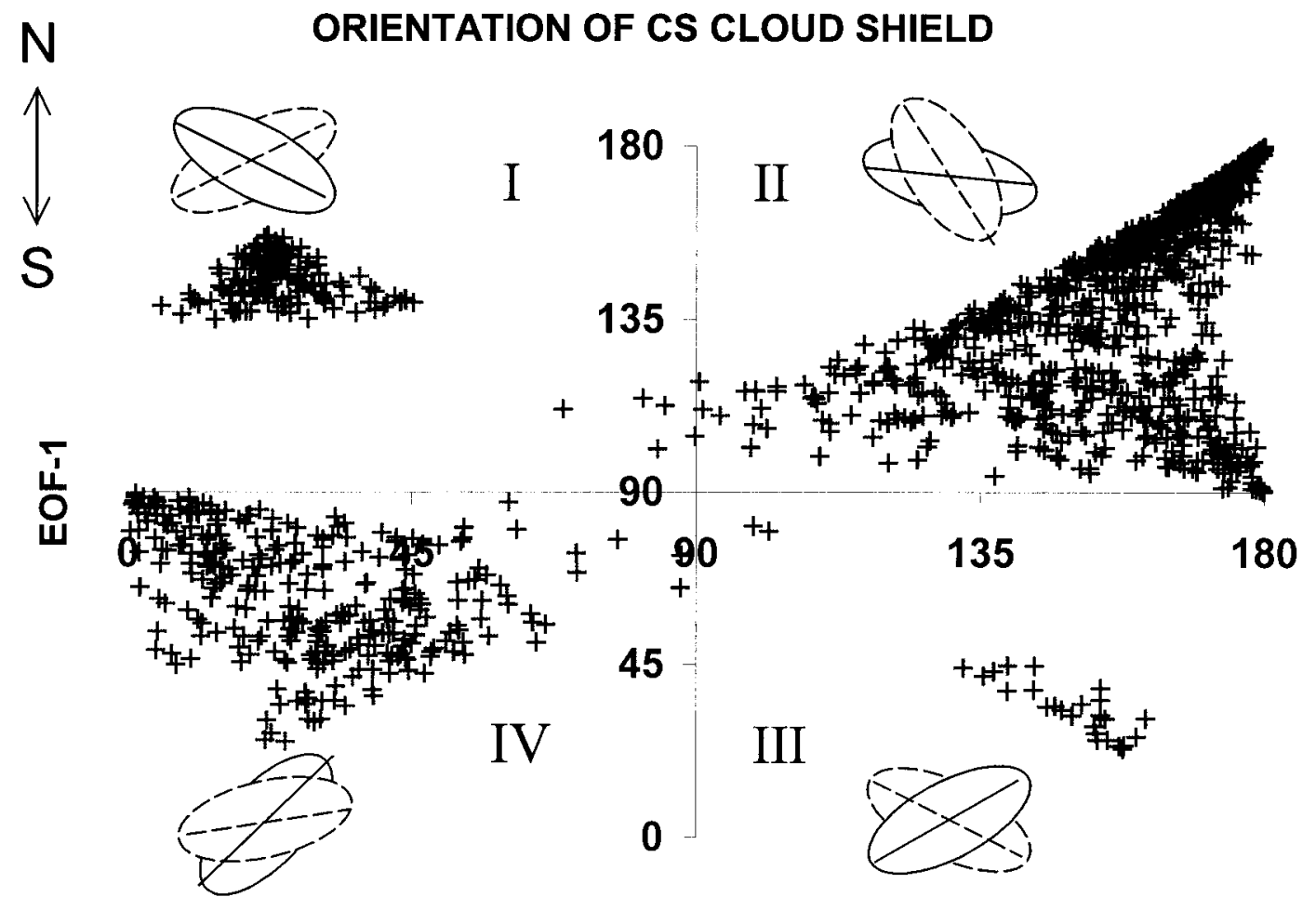

LEAST SQUARES

FIG. 8. Comparison between the orientation of CS cloud shield with two methodologies: EOF1 and least squares. Ellipses are sketched to illustrate the differences associated with each quadrant (Roman numbers) in the dispersion diagram. Dashed ellipses represent least squares, and solid line ellipses are the EOF1 orientation.

low the observation of the very beginning of the CS life cycles, on the other hand, it is representative of important properties of the mesoscale convective activity.

During the 7-day case studies, the largest majority of CS prevailing direction of displacement showed negative (east to west) zonal component and negative meridional component (north to south). The easterly displacement of the CS is consistent with the dominant low-level easterly winds during that period. This observation is based on the daily composite of the 700hPa National Centers for Environmental Prediction-National Center for Atmospheric Research (NCEP-NCAR) reanalysis (not shown). Even considering that some CS exhibited long displacements, the spatial pattern of the CS life cycles still suggests the characteristic banded pattern shown in Garreaud and Wallace (1997).

\section{b. Monitoring of structural properties of the CS}

The orientation of squall-line cloud tops in the Amazon basin using IR images has been investigated in both observational analysis (Cohen et al. 1995) and model simulations (Silva Dias and Ferreira 1992). An accurate and automated method to identify squall-line orientation allows, among other applications, objective rather than subjective analysis of case studies. The hor- izontal orientation of the first eigenvector (EOF1) incorporated in the MASCOTTE method provides another way to infer the main orientation of the cloud shield of the CS. The orientation is computed as the counterclockwise angle between the east-west direction and the direction of the first eigenvector. Therefore, the orientation angle is $0<\mathbf{E O F} 1 \leq 180^{\circ}$. For example, an orientation angle equal to $135^{\circ}$ implies an orientation northwest-southeast. The same convention is used for the least squares (LS) method. Figure 8 shows the dispersion diagram obtained with the LS and EOF1 orientations. Ellipses are sketched to exemplify the meaning of the dispersion in each quadrant of Fig. 8. For instance, in quadrant I, the EOF1 method (full-line ellipses) indicates a NW-SE orientation of the CS whereas LS (dashed-line ellipses) gives a NE-SW orientation. The opposite situation is observed in quadrant III. In quadrant II, both methods prescribe NW-SE orientations. Likewise, in quadrant IV, the LS and EOF1 methods indicate NE-SW orientations. Note that the largest discrepancies between both methods relative to the north-south direction occur in quadrants I and III. Quadrants II and IV indicate the wide range of dispersion, although in these cases both methods ascribe the CS orientation in the same quadrant relative to the northsouth direction. A more detailed discussion about the 
accuracy and differences between both methods will be shown with examples in section 4.

An important parameter that characterizes the structural properties of CS is the eccentricity of the system. It has been used to characterize mesoscale convective complexes (MCC) (Maddox 1980; Velasco and Fritch 1987). According to the definition of Ecc assumed by MASCOTTE (i.e., Ecc $=\|\mathbf{E O F 2}\| / \|$ EOF1 $\|$ ), the values of Ecc are in the interval $0<$ Ecc $\leq 1$. Thus, high (low) magnitudes of Ecc are associated with CS shapes that are circular (linear). For that reason, an accurate methodology to determine orientation of the axis with maximum and minimum variation of CS cold pixels is crucial for the estimation of Ecc. Although eccentricity has been used as a key property to identify typical characteristics of squall lines (Cohen et al. 1995) and MCCs (Maddox 1980), it does not account for the collapsing and fragmentation of cold cloud tops that very often occur as an MCS evolves (e.g., Rickenbach 1999). One simple and efficient approach to quantify fragmentation is developed in MASCOTTE with the use of the perimeter-area relationship. Lovejoy (1982) showed that the representation of logarithm of perimeters versus the logarithm of area of the systems has approximately a linear relationship, which apparently holds for several spatial scales. The slope of the straight line in this relationship was first defined by Lovejoy (1982) as the fractal dimension of clouds and rain. However, not all fluctuations of perimeters of clouds and rain can be explained by monofractal models. In fact, this early idea of monofractality has been replaced by the universal multifractal concept [see Lovejoy and Schertzer (1990) for further details]. This original concept of Lovejoy (1982) is indirectly used in MASCOTTE to measure fragmentation of clouds. The procedure in this case is to remove the trend between perimeter and area, by assuming the following functional relationship:

$$
\log _{10}(\text { perimeter })=\left[\text { const }+\beta \log _{10}(\text { area })\right]+\text { Fluct },
$$

where const and $\beta$ are the linear and angular coefficients, respectively. Fluct is the residue and can be interpreted as the fluctuation of the logarithm of perimeter that does not account for the area of cloud tops. In other words, after extracting the functional dependence of area on perimeter, the residue is the fluctuation of perimeter that is due only to changes in fragmentation or increase/ decrease of irregularities of the boundaries of CS cold tops. Therefore, positive magnitudes of Fluct are associated with relative increases of fragmentation of clouds, whereas negative magnitudes of Fluct are indicative that the boundaries of cold shields are more regular or less fragmented. Together with eccentricity, the Fluct parameter can be applied to characterize different stages of development and decaying of CS.

According to the conceptual model of cloud shield evolution of CS discussed in Rickenbach (1999), cold cloud anvils of propagating squall lines under direc- tional vertical wind shear are subject to tilting and displacement of the leading edge. This model hypothesizes that as the deep cells develop they "tilt" to the direction of the upper-level winds, opposite to direction of propagation of the MCS precipitation pattern. As the leading edge of the convective precipitation weakens, the coldest portion of the anvil, located where convection had been deepest, becomes separated from the leading edge as the anvil continues to spread to the opposite direction of the storm propagation. From the point of view of the satellite imagery, this displacement of the cold cloud shield would be characterized by an apparent sink of the cold cloud top and, therefore, an increase of $T_{B}$. The consequent apparent displacement of the cold shield and warmth of portions of the cloud anvil would increase the fragmentation or irregularity in the form of a CS. Although the Rickenbach (1999) hypothesis was postulated for strong directional shear, it is also possible that the vertical speed shear can cause a similar effect on the cloud tops.

To verify the consistence between the general fragmentation of CS cloud shield and the absolute vertical speed shear (AVSS), we computed the diurnal variation of AVSS between 700 and $200 \mathrm{hPa}$ (Fig. 9). This computation was performed with the NCEP-NCAR reanalysis in central Amazon. The area of investigation extended from $15^{\circ} \mathrm{S}$ to $5^{\circ} \mathrm{N}$ and $67.5^{\circ}$ to $34.0^{\circ} \mathrm{W}$ with the purpose of excluding the Andes. Figure 9 indicates a clear diurnal cycle of AVVS, with a maximum around 1800 UTC (1200 LT) and a minimum around 0600 UTC (0200 LT). The maximum and minimum occurred within the period of increasing and decreasing number of $\mathrm{CS}$, respectively (Fig. 6). Figure 10 shows the distribution of Fluct around the time of maximum and minimum AVSS. During the time of maximum AVSS (1845 UTC), $65 \%$ of CS are associated with positive Fluct, which is indicative of enhancement in CS fragmentation. On the other hand, at the time of minimum AVSS (0645 UTC), only $41.8 \%$ of CS fell in positive Fluct categories. Although CS could have been observed in any stage of development during these two periods, the environment in which they were propagating may have affected their structural aspect. The monitoring of the distribution of Fluct can alternately be useful to distinguish CS properties in different large-scale wind profile regimes.

\section{c. Monitoring of CS with 2-3-h satellite image intervals}

An important effort in the elaboration of MASCOTTE has been to extend its applicability to the broad options of geostationary satellite data presently available. The International Satellite Cloud Climatology Project (ISCCP), for instance, has continuously provided a unique source of calibrated and uniformly formatted geostationary and polar-orbiting weather satellite data for climate studies (Schiffer and Rossow 1983). The infrared $(11 \mu \mathrm{m})$ and visible $(0.6 \mu \mathrm{m})$ datasets (ISCCP- 


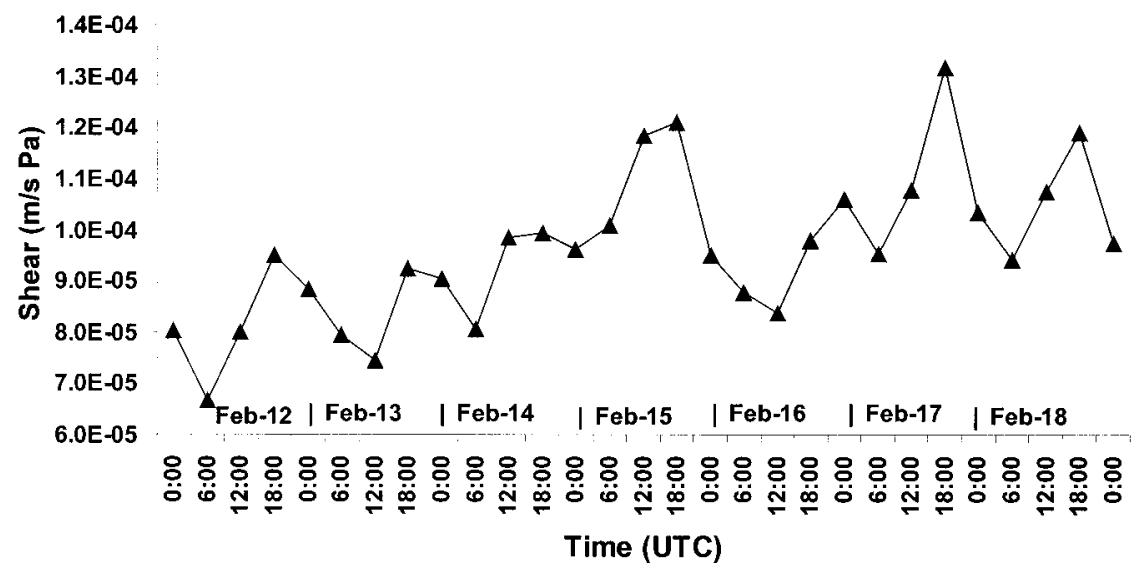

FIG. 9. Diurnal variation of the absolute vertical speed shear between 700- and 200-hPa levels from 0000 UTC 12 Feb to 0000 UTC 19 Feb. Days are indicated between bars on the abscissa. Data source: NCEP-NCAR reanalysis.

B3) represent the original satellite images sampled at intervals of $3 \mathrm{~h}$ and $30 \mathrm{~km}$ and have been used in many studies for tracking CS in tropical areas (Garreaud and Wallace 1997; Machado et al. 1998).

To test the use of MASCOTTE when the time interval between satellite images is greater than $1 \mathrm{~h}$, we compared our previous analysis with the same set of GOES images but with 2 - and 3-h time intervals. The results indicate that the criterion of maximum spatial correlation still holds for these time intervals, although the magnitudes of $r_{s}$ evidently decrease. A good correspondence between CS life cycles with different coarse time resolutions ( 2 and $3 \mathrm{~h}$ ) was obtained, but with some discrepancy in the total number of splits and merges. Overall, the results support the application of MASCOTTE for coarse time intervals between satellite images.

\section{Monitoring of individual CS life cycles}

The objective of this section is to discuss the skill of MASCOTTE for the automated tracking of CS life cycles and determination of their structural properties. With this purpose in mind, we describe the complete life cycle of a CS that evolved on 15 February (Fig. 11). This example is selected to illustrate the determination of merges and splits, which are crucial to determine the correct characterization of the CS propagation properties. MASCOTTE first detected the CS (hereinafter referred as $\mathrm{CS}-\mathrm{a}$ ) that matched the $T-R$ criteria at 0745 UTC. High (cold) cloud clusters (CC) were embedded in the east-west orientation of the CS-a. At 0845 UTC, as CS-a slowly propagated southwestward, the coldest $\mathrm{CC}$ increased their size with a preferable enhancement to the east of the CS-a. At 0945 UTC, merging with another CS was observed and detected by MASCOTTE. As previously explained, the merging is detected because of the increase in the CS area and decrease of spatial correlation $r_{s}$ between two consec- utive images. A new CS was then defined and is hereinafter referred to as CS-b. One hour after the merge, the cloud shield of CS-b continued to expand while the fraction of CC decreased to the west of CS-b. The consequent sinking and warming to $T_{B}>235 \mathrm{~K}$ of these decaying $\mathrm{CC}$ were clearly observed at 1145 UTC. One hour later, CS-b continued to decay, and the elongated east-west appearance of the warmer isotherms changed to a more circular shape. As the trailing anvil of CS-b decayed, new cells at the center of the CS-b continued to propagate slowly southwestward, as indicated at 1345 and 1445 UTC. The propagation of new CC southwestward and the sinking of the trailing anvil defined a new spatial configuration of CS-b with a near-northeast-southwest orientation of the axis of maximum variability. At 1545 UTC, the fraction of CC continued to increase to the left and right of the southwestward direction of propagation of $\mathrm{CS}-\mathrm{b}$, with much higher development of the left cell. The splitting of CS-b was detected by MASCOTTE at 1645 UTC because of the presence of two CS with positive $r_{s}$ (hereinafter referred as CS-c and CS-d). The maximum correlation with CS$\mathrm{b}$ at 1545 UTC occurs with the larger CS at 1645 UTC (defined as CS-c), which corresponded to the left CC relative to the mean direction of propagation of CS-b. MASCOTTE determined the center of gravity of CS-c and CS-d at 1645 UTC, allowing the continuous tracking of each individual CS. CS-d decayed in the next hour, and MASCOTTE no longer detected it. The nearly northwest-southeast orientation of CS-c cloud shield at 1745 UTC corresponded to a shift of $90^{\circ}$ relative to CSb from 0945 to 1145 UTC. The elongated pattern of CS-c became more evident after 1745 UTC, as new CC continuously developed south of CS-c. At 1845 UTC, CS-c probably evolved into a well-defined squall line identified in IR satellite images. One hour later, new splitting followed by merging with a large CS occurred, and the configuration of CS-c drastically changed (not 

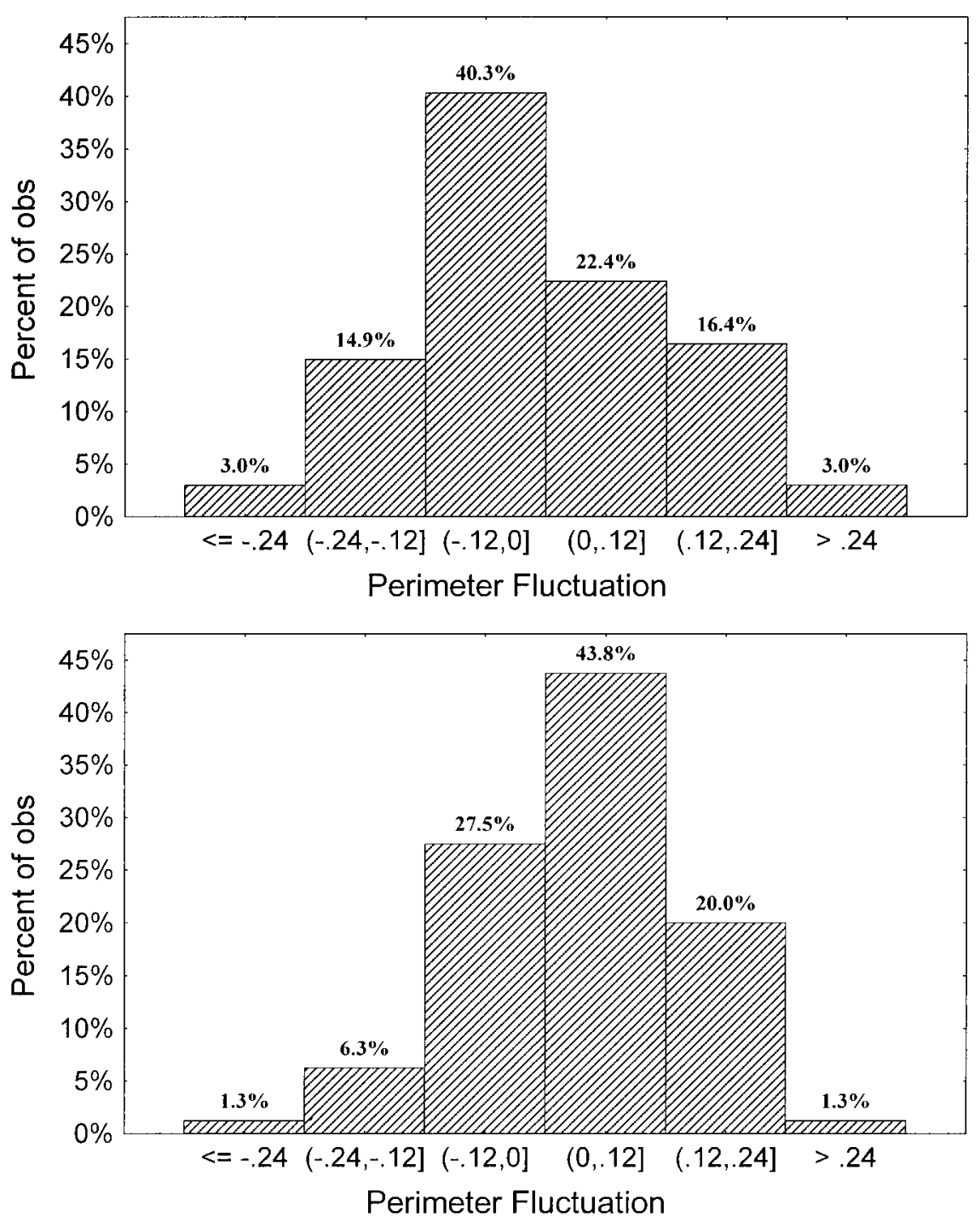

FIG. 10. Distribution of perimeter fluctuation (Fluct) around the times of (bottom) maximum (1845 UTC) and (top) minimum (0645 UTC) absolute vertical speed shear for 12-18 Feb. Fluct standard deviation $=0.12$.

shown). The tracking of this new CS by MASCOTTE indicated that after merging its life cycle extended into the next day (16 February).

Supplementary information on the structural properties of CS-b and CS-c, as determined by MASCOTTE, is summarized in Fig. 12. For each time, MASCOTTE displays the perimeter of the CS, the ellipses whose axes are determined by EOF1 and EOF2, and the eccentricity. In addition, the CS orientation determined by the least squares fit (dashed line) as defined in Machado et al. (1998) is shown for the sake of comparison with the MASCOTTE method. Time (UTC), eccentricity (Ecc), equivalent radius $(R)$, fraction of clouds with $T_{B} \leq 210$ $\mathrm{K}(\mathrm{Fc})$, and speed and direction (counted as clockwise angle from north) of propagation of CS are indicated in each panel. For display purposes, the center of gravity (cg) of the CS is collocated with the center of the image. The ordinate and abscissa are in arbitrary units of latitude and longitude, respectively. We recall that the speed is computed by MASCOTTE only when no merging or splitting is observed in the CS.

At 0945 UTC, when CS-b was first determined after a previous merging, $\mathrm{Fc}$ was the largest of its entire life cycle. Eccentricity is low (0.29) because of the elongated nature of the squall line. At 1045 UTC, the equivalent radius and eccentricity of CS-b increased, whereas the fraction of cold cloud tops decreased. The decrease of $\mathrm{Fc}$ was indicative of decaying in the northwestern portion of the CS. The propagation speed of CS-b from 0945 to 1045 UTC was approximately $31 \mathrm{~km} \mathrm{~h}^{-1}$, and direction was $71^{\circ}$ (easterly propagation). At 1145 UTC, large decrease in the relative radius is observed, with 

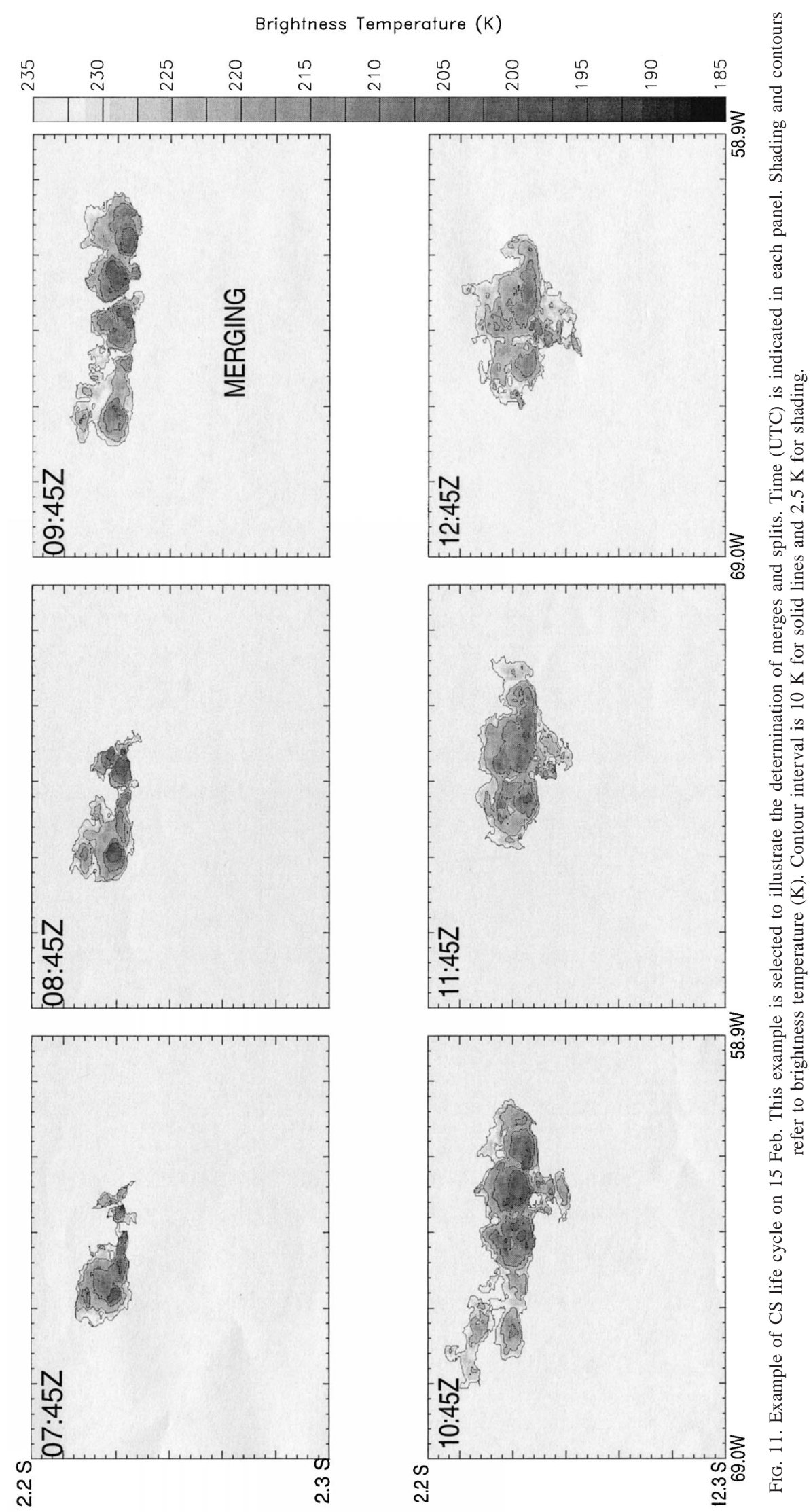
Brightness Temperature (K)

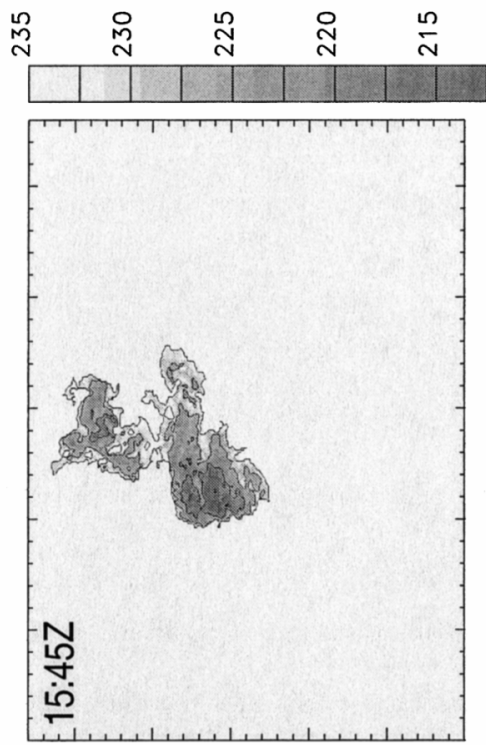

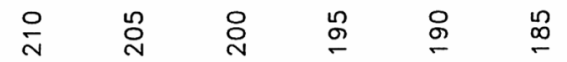
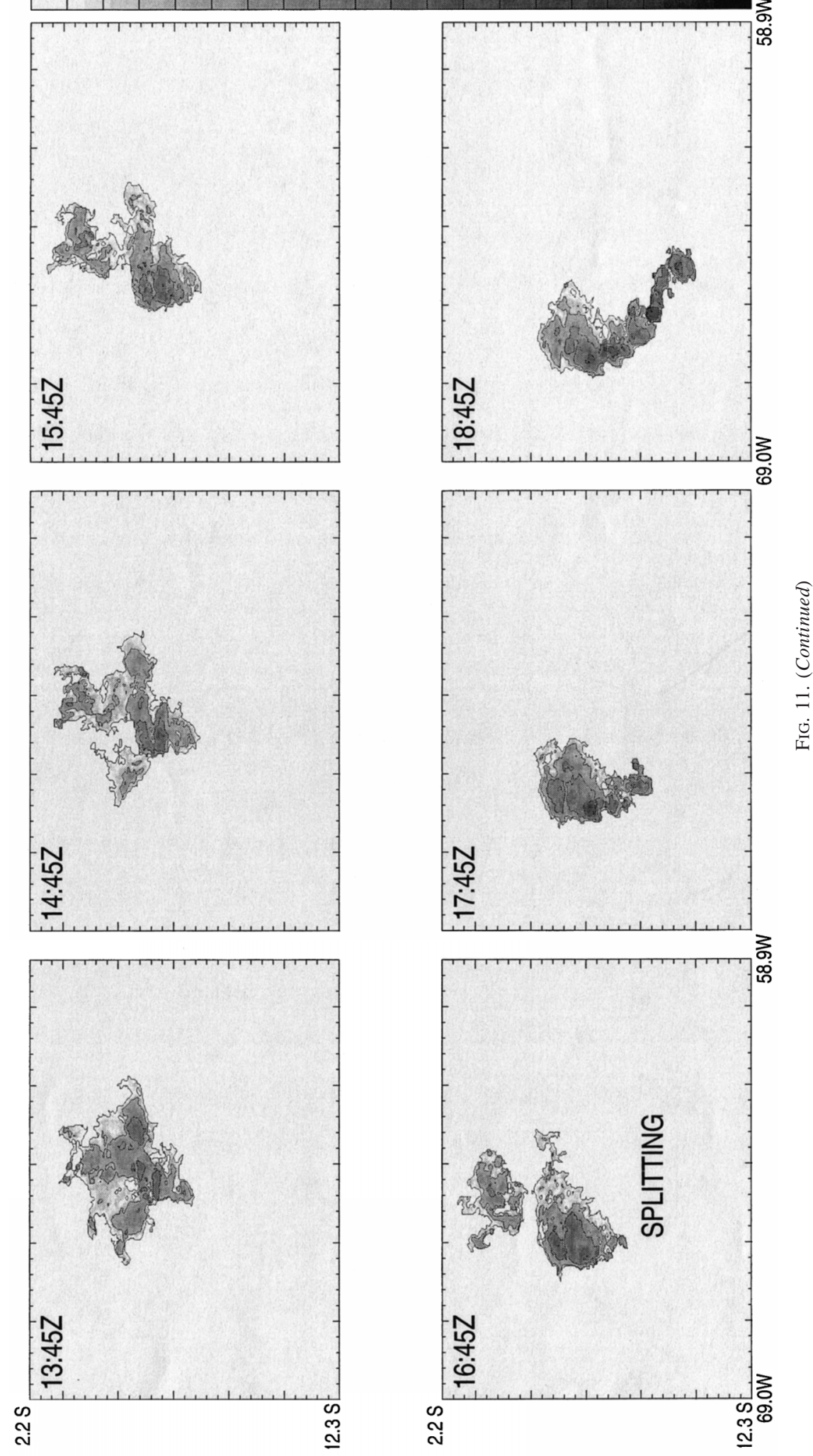

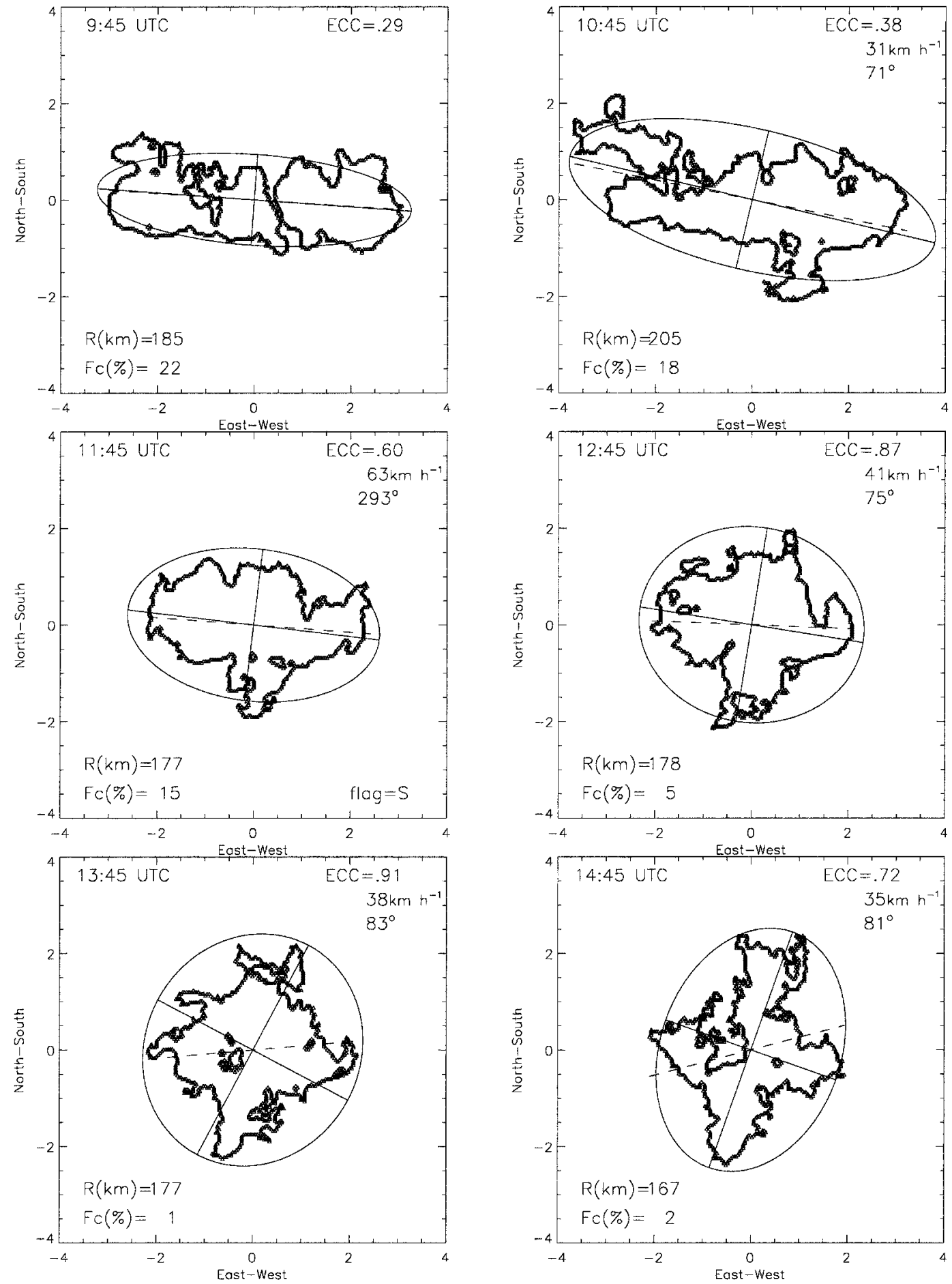

FIG. 12. Example of structural properties of the same CS life cycle discussed in Fig. 11. For each time (UTC) the panels display the perimeter, the ellipses whose axes are determined by the first and second eigenvectors EOF1 and EOF2, and the eccentricity. Also shown are the eccentricity (Ecc), equivalent radius $(R)$, fraction of clouds $(\mathrm{Fc})$ with $T_{B} \leq 210 \mathrm{~K}$, and speed and direction (counted as clockwise angle from north) of propagation of CS. The CS orientation determined by the least squares fit is indicated as dashed lines. The center of gravity (cg) of the CS is collocated with the center of the image. Flag equal to $\mathrm{S}(\mathrm{M})$ indicates possible sinking/splitting (merging) of CS with $R<100 \mathrm{~km}$. The ordinate and abscissa are in arbitrary units of lat and long, respectively. 

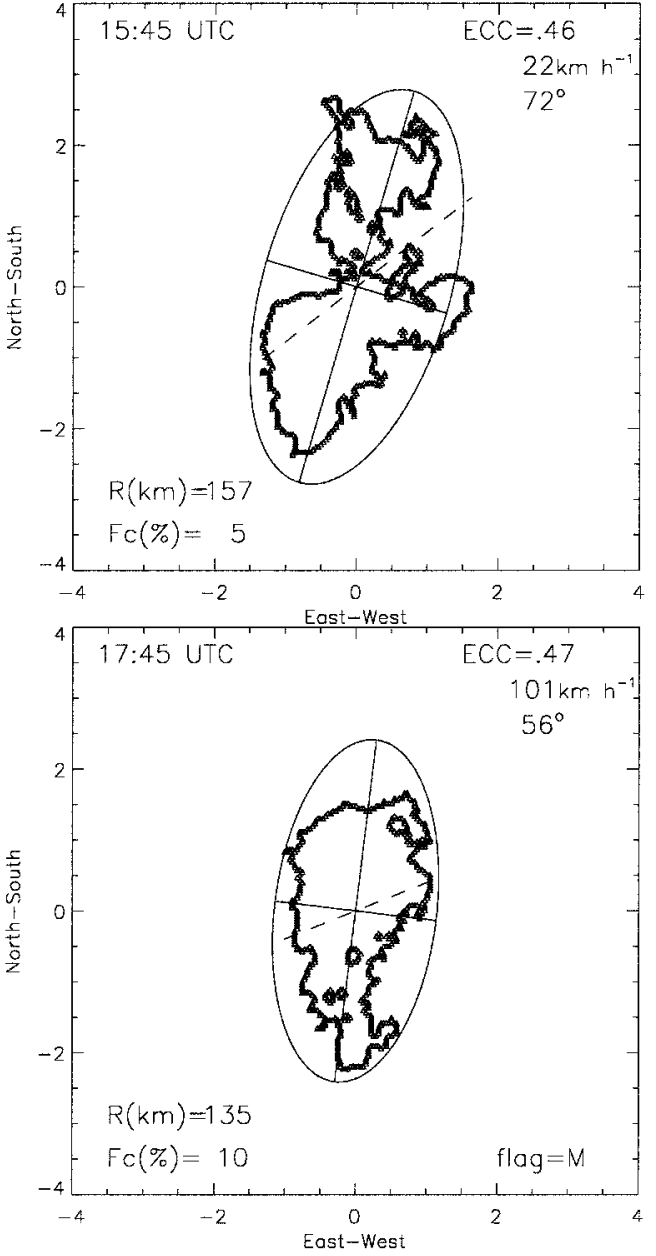
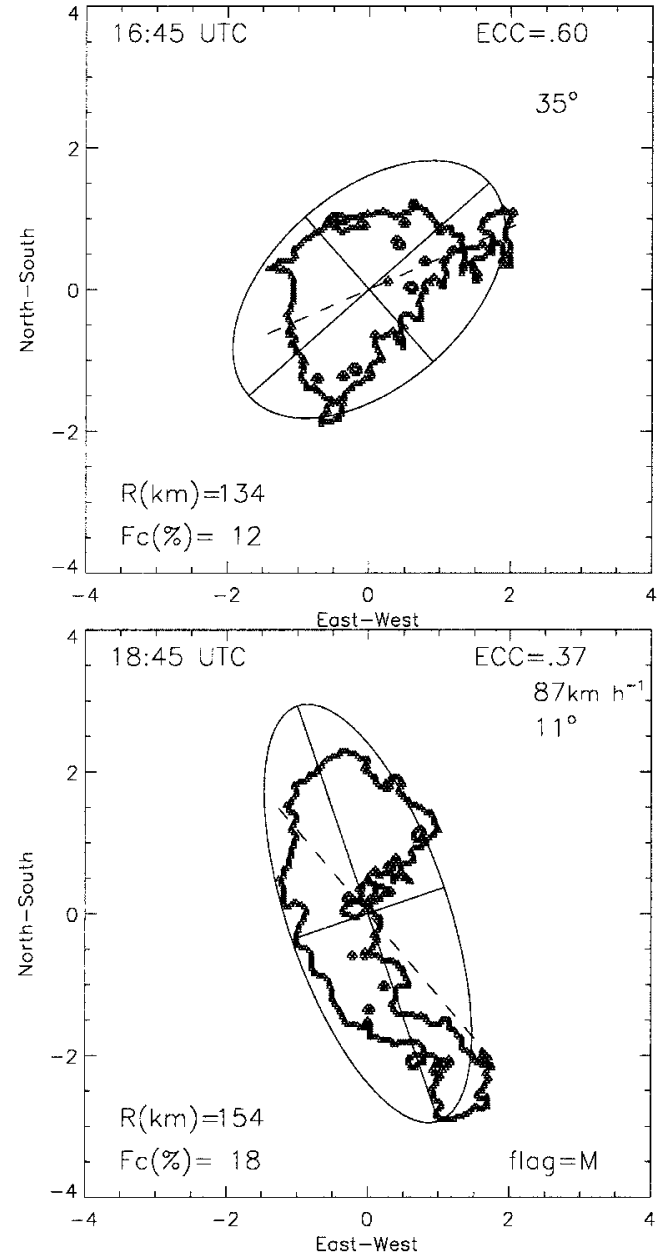

FIG. 12. (Continued)

some sinking and decaying of $\mathrm{CC}$ on the right side relative to the direction of propagation of the CS-b. Because of this dramatic change in the total area of the CS-b, the propagation speed and the magnitude of the meridional component also increased. This result suggests translation of cg toward the left side $\mathrm{CC}$ relative to the direction of propagation of the CS-b. One hour later (1245 UTC), $R$ remained practically the same, whereas Fc decreased and Ecc increased, indicating the decaying phase of the CS-b. The speed and direction of propagation showed westward movement of the CS with speed of $41 \mathrm{~km} \mathrm{~h}^{-1}$. After $1345 \mathrm{UTC}, \mathrm{CS}-\mathrm{b}$ began to collapse and became more fragmented as the old and mature $\mathrm{CC}$ decayed on the right side of the propagation. At the same time, the development of new $\mathrm{CC}$ on the left side modified the spatial pattern of CS-b. The change in the orientation of CS-b cloud tops after 1445 UTC from east-west to northeast-southwest is very noticeable. These changes were properly captured by the rotation of EOF1. Furthermore, it is evident that the least squares method of Machado et al. (1998) misrepresents the actual orientation of cloud shield when the shape of the CS became more irregular (from 1345 through 1845 UTC). The least squares approach is consistent with the MASCOTTE EOF1 method only when the boundaries of CS were more regular. This is the case independently whether the CS are more elongated or are circular (compare 0945 and 1245 UTC). Changes in the orientation of EOF1 and in the fragmentation of the CS did not affect the eastward direction of propagation of the CS-b determined by the translation of cg, until 1545 UTC when splitting was observed. The splitting of CS-b and consequent displacement of CS-c (left side relative to the direction of propagation) led to an increase in the magnitude of the meridional component of the direction of propagation of CS-c at 1645 UTC. The splitting was followed by an enhancement of new CC south of CS-c and rotation of EOF1 toward north as shown in the images at 1745 UTC (Figs. 10 and 11). At 1845 UTC, a rapid increase (approximately $30 \%$ ) in area associated with an increase of speeds of more than 2 times the average value observed for the entire life cycle suggested that CS-c merged with another convective system with $R$ less than $100 \mathrm{~km}$ (compare the 


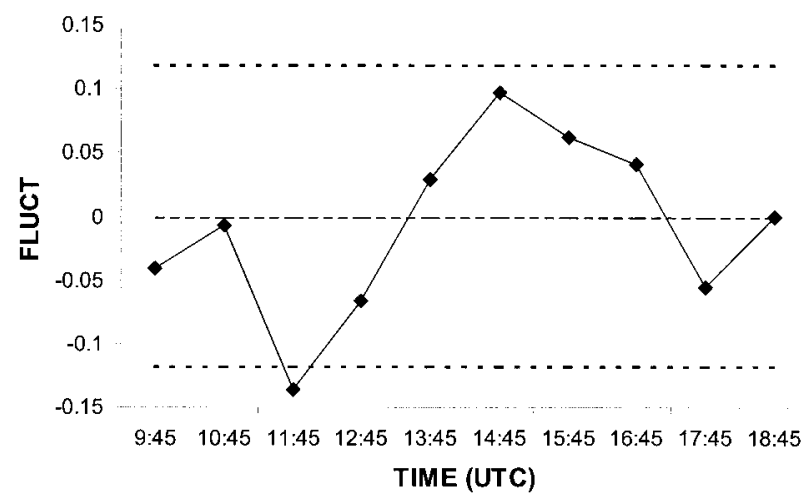

FIG. 13. Time evolution of fragmentation parameter Fluct for CS-b (from 0945 to 1545 UTC) and CS-c (from 1645 to 1845 UTC).

south boundary of CS-c at 1745 and 1845 UTC). CS$c$ at 1845 UTC was associated with a simultaneous increase of area and $r_{s}$. However, a flag for merging was set by MASCOTTE because of the relevant rate of change in structural properties (perimeter and area) and dynamical properties (speed, propagation direction, and EOF1 orientation), which were likely related to a merge with a relatively small convective system.

One important aspect of the structural properties of evolving CS is the distinction of collapsing stages of the cloud shield. Figure 13 shows the time evolution of Fluct for CS-b (from 0945 to 1545 UTC) and CS-c (from 1645 to 1845 UTC). The transition of Fluct from the lowest magnitude at 1145 UTC to the largest magnitude at 1445 UTC is noticeable. An inspection of CS-b in this time interval (Fig. 12) clearly indicates the reasons for that transition: the area of CS-b remains almost the same whereas the perimeter increases as the anvil shield collapses. It is interesting to note the independence between Fluct and Ecc. This example visibly emphasizes that any automated method for tracking of CS needs a definition of a parameter to account for fragmentation of the system in addition to its eccentricity. Relatively large magnitudes of Fluct are observed until 1545 UTC, whereas the fragmentation of CS-c decreases after splitting. At 1845 UTC, with the development of new cells south of CS-c, the spatial configuration becomes more similar to CS-b at 1045 UTC, regardless of differences in area and orientation. Therefore, the simultaneous monitoring of $R, \mathrm{Fc}, \mathrm{Ecc}$, and Fluct is extremely important to design an accurate satellite method to identify convective stages of evolving CS.

\section{Summary and conclusions}

A simple, fully automated, and efficient method to determine the structural properties and evolution (tracking) of CS cloud shields has been described. This method, which is based on the maximum spatial correlation tracking technique, is a new alternative to the existent techniques available for studies that monitor the evo- lution of CS using satellite images. MASCOTTE provides as CS structural properties the following parameters: horizontal area, perimeter, mean and variance of brightness temperature, minimum brightness temperature, fractional convective area, and center of gravity. A new way of estimating the orientation and eccentricity of CS is proposed that is based on EOF analysis of CS pixel coordinates. Based on the magnitudes of the spatial correlation between consecutive satellite images and the changes in horizontal areas of CS, MASCOTTE provides a new way of tracking the evolution of CS life cycles. This method is a significant improvement over previously published methods that assume specific geometries to determine the CS propagation (Machado et al. 1998). MASCOTTE has also proven to be useful for monitoring the CS fragmentation and accurately determining the CS cloud-top orientation. Moreover, MASCOTTE provides automated determination of the exact occurrence of splits and merges of CS, even for time intervals between satellite images greater than $1 \mathrm{~h}$. An important advantage of the MASCOTTE method is the visual identification and checking of the CS evolution as the algorithm is applied to satellite images. The versatility of MASCOTTE allows application to any extensive satellite imagery database and any geographic region.

The focus of this paper has been on describing the MASCOTTE methodology and applying it to seven consecutive days (12-18 February) during the WETAMC LBA-TRMM campaign over the Rondônia state in the Amazon basin. Convective systems in the Amazon have a high degree of complexity and interaction with the large-scale circulation. For instance, during the WETAMC, Silva Dias and Zipser (1999, personal communication) have observed periods of suppressed organized convection due to the synoptic-scale regime being dominated by the migration of the Brazilian northeast upper-level trough. This is the same regime observed during the dry season, which is typically ascribed to a much lower frequency of organized synoptic-scale systems. The significance of the many possible feedbacks among clouds, rainfall, and surface processes over the Amazon basin needs to be further understood. Studies that are based on satellite data composites (e.g., Garreaud and Wallace 1997) do not resolve the CS variability, especially the diurnal cycle and geographic distribution. Satellite tracking methods such as the one described here provide an additional research tool in this effort. Statistical analysis of the occurrence of different CS structural properties during varying synoptic regimes can provide valuable information on the interaction between CS and the large-scale circulation over the Amazon.

Acknowledgments. The authors greatly appreciate many helpful discussions with Maria Assunção F. da Silva Dias, Tom Rickenbach, and Luis A. T. Machado. The availability of GOES satellite images at the NASA 
Goddard Space Flight Center as part of the LBATRMM field campaign was very helpful. Leila M. V. de Carvalho is grateful for the financial support from Fundação de Amparo à Pesquisa do Estado de São Paulo (FAPESP Project 98/14414-0). Partial support from the Jet Propulsion Laboratory (JPL) for Charles Jones (Subcontract 961593) is also acknowledged.

\section{REFERENCES}

Carvalho, L. M. V., 1998: Mesoscale cloud patterns in satellite images: Use of fractal dimensions and statistical multivariate analysis (in Portuguese). Ph.D. thesis, Dept. Atmospheric Sciences, University of São Paulo, São Paulo, Brazil, 264 pp.

Chen, S. S., and R. A. Houze, 1997: Diurnal variation and life-cycle of deep convective systems over the tropical Pacific warm pool. Quart. J. Roy. Meteor. Soc., 123, 357-388.

,$- \ldots$, and B. E. Mapes, 1996: Multiscale variability of deep convection in relation to large-scale circulation in TOGA COARE. J. Atmos. Sci., 53, 1380-1409.

Cohen, J., M. A. F. Silva Dias, and C. Nobre, 1995: Environmental conditions associated with Amazonian squall lines: A case study. Mon. Wea. Rev., 123, 3163-3174.

Cotton, W. R., and R. A. Anthes, 1989: Storm and Cloud Dynamics. Academic Press, 880 pp.

Endlich, R. M., and D. E. Wolf, 1981: Automatic cloud tracking applied to GOES and METEOSAT observations. J. Appl. Meteor., 20, 309-319.

Garreaud, R. D., and J. M. Wallace, 1997: The diurnal march of convective cloudiness over the Americas. Mon. Wea. Rev., 125, 3157-3171.

Houze, R. A., Jr., 1977: Structure and dynamics of a tropical squallline system. Mon. Wea. Rev., 105, 1540-1567.

_ 1993: Cloud Dynamics. Academic Press, 570 pp.

— , and P. V. Hobbs, 1982: Organization and structure of precipitating cloud systems. Advances in Geophysics, Vol. 24, Academic Press, 225-315.

Jackson, J. E., 1991: A User's Guide to Principal Component Analysis. Wiley-InterScience, $569 \mathrm{pp}$

Johnson, R. H., W. A. Gallus Jr., and M. D. Vescio, 1990: Neartropopause vertical motion within the trailing-stratiform region of a midlatitude squall line. J. Atmos. Sci., 47, 2200-2210.

Lovejoy, S., 1982: The area-perimeter relationship for rain and cloud areas. Science, 216, 185-187.

—, and D. Schertzer, 1990: Multifractal, universal classes and sat- ellite and radar measurements of clouds and rain. J. Geophys. Res., 95, 2021-2034.

Machado, L. A. T., and W. B. Rossow, 1993: Structural characteristics and radiative properties of tropical cloud clusters. Mon. Wea. Rev., 121, 3234-3260.

_, M. Debois, and J. P. Duvel, 1992: Structural characteristics of deep convective systems over tropical Africa and Atlantic Ocean. Mon. Wea. Rev., 120, 392-406.

—_ W. B. Rossow, R. L. Guedes, and A. W. Walker, 1998: Life cycle variations of mesoscale convective systems over the Americas. Mon. Wea. Rev., 126, 1630-1654.

Maddox, R. A., 1980: Mesoscale convective complexes. Bull. Amer. Meteor. Soc., 61, 1374-1387.

Mapes, B. E., and R. A. Houze Jr., 1993: Cloud clusters and superclusters over the oceanic warm pool. Mon. Wea. Rev., 121, 13981415.

Miller, D., and J. M. Fritsch, 1991: Mesoscale convective complexes in the western Pacific region. Mon. Wea. Rev., 119, 2978-2992.

Mohr, K. I., and E. J. Zipser, 1996: Mesoscale convective systems defined by their $85-\mathrm{GHz}$ ice scattering signature: Size and intensity comparison over tropical oceans and continents. Mon. Wea. Rev., 124, 2417-2437.

Nobre, C. A., and Coauthors, 1996: The Large-Scale BiosphereAtmosphere Experiment in Amazonia (LBA) concise experiment plan. LBA Science Planning Group Rep., 44 pp. [Available from Institute of Hydrology, Wallingford, Oxfordshire, United Kingdom.]

Rickenbach, T. M., 1999: Cloud top evolution of tropical oceanic squall lines from radar reflectivity and infrared satellite data. Mon. Wea. Rev., 127, 2951-2976.

Shiffer, R. A., and W. B. Rossow, 1983: The International Satellite Cloud Climatology Project (ISCCP): The first project of the World Climate Research Program. Bull. Amer. Meteor. Soc., 64, 779-784.

Silva Dias, M. A. F., and R. N. Ferreira, 1992: Application of a linear spectral model to the study of Amazonian squall lines during GTE/ABLE 2B. J. Geophys. Res., 97, 20 405-20 419.

—_ and Coauthors, 2000: Rainfall and surface processes in Amazonia during the WETAMC/LBA: An overview. Preprints, Sixth Int. Conf. on Southern Hemisphere Meteorology and Oceanography, Santiago, Chile, Amer. Meteor. Soc., 249-250.

Velasco, I., and J. M. Fritsch, 1987: Mesoscale convective complexes in the Americas. J. Geophys. Res., 92, 9591-9613.

Williams, M., and R. A. Houze, 1987: Satellite-observed characteristics of winter monsoon cloud clusters. Mon. Wea. Rev., 115, 505-519.

Zipser, E. J., 1988: The evolution of mesoscale convective systems: Evidence from radar and satellite observations. Tropical Rainfall Measurements, J. S. Theon and N. Fugono, Eds., Deepak, 159166. 